\title{
SHAPE SENSITIVITIES FOR AN INVERSE PROBLEM IN MAGNETIC INDUCTION TOMOGRAPHY BASED ON THE EDDY CURRENT MODEL*
}

\author{
MICHAEL HINTERMÜLLER ${ }^{\dagger}$, ANTOINE LAURAIN ${ }^{\ddagger}$, AND IRWIN YOUSEPT ${ }^{\S}$
}

\begin{abstract}
In this paper the shape derivative of an objective depending on the solution of an eddy current approximation of Maxwell's equations is obtained. Using a Lagrangian approach in the spirit of Delfour and Zolésio, the computation of the shape derivative of the solution of the state equation is bypassed. This theoretical result is applied to magnetic impedance tomography, which is an imaging modality aiming at the contactless mapping (identification) of the unknown electrical conductivities inside an object given measurements recorded by receiver coils.
\end{abstract}

Key words. magnetic induction tomography, inverse problems, shape optimization, shape derivative

AMS subject classifications. Primary 49N45,35Q61; Secondary 47J30, 49Q10

1. Introduction. Magnetic induction tomography (MIT) is a low-contrast resolution mapping modality for non-contacting measurement of electric properties of conducting materials which has been developed for industrial processes about twenty years ago and whose use is more recent in medical imaging; see $[12,17,26,27,30]$. The technology of MIT involves an oscillating magnetic field generated by a transmitter coil, which in turn induces an eddy current inside the conducting materials. The resulting magnetic field arising from the eddy current depends on the conductivity distribution in the region surrounded by the coil array and it is detected by a set of receiver coils. Compared to other imaging techniques such as electrical impedance tomography, the advantage of MIT is to avoid electrode-skin contact since the magnetic field can penetrate through the insulating barrier, making it a contactless and non-invasive technique. The goal of this paper is to study an inverse problem of a simplified MIT system governed by the time-harmonic eddy current equations. In fact, given measurements, which depend on the electric field $\mathbf{E}$ and the current density $\mathbf{J}$ and which are recorded at receiver coils surrounding the region of interest, we aim at reconstructing the conductivity (function) $\sigma$ associated with objects contained in the region of interest.

Eddy current equations play a significant role in many advanced technologies involving electromagnetic phenomena. These equations arise from Maxwell's equations by neglecting the presence of displacement currents. In a low frequency range, they provide a reasonable approximation of the full Maxwell equations; see [2]. For recent results on the mathematical and numerical analysis of the optimal control of eddy current equations, we refer to [24,35,40-42]. The use of the eddy current model in MIT is justified by the small wavelength of its operating frequencies. Typically, the frequencies lie between 10 and $100 \mathrm{MHz}$, i.e. in the range of some micrometers, so that the wavelength is small compared to the size of the conductor.

The inverse problem of MIT is ill-posed, in the sense that, besides other structural difficulties, there is no uniqueness and stability of the solution. In many practical applications, this may be exacerbated by the small number of available measurements and by low regularity of the hidden objects: typically the associated (conductivity) functions are bounded but not continuous. A few algorithms have been proposed to solve the inverse problem in two dimensions or in the discrete setting; see [10,31,32,37,39] and references therein.

In order to deal with the ill-posedness in numerical studies, one often introduces a regularization in the reconstruction process. A widely used solution approach to inverse problems consists in minimizing

\footnotetext{
*M.H. acknowledges support by the Austrian Science Fund FWF under SFB F32-N18 "Mathematical Optimization and Its Applications in Biomedical Sciences

${ }^{\dagger}$ Humboldt-Universität zu Berlin, Department of Mathematics, Unter den Linden 6, 10099 Berlin, Germany (hint@math.hu-berlin.de)

${ }^{\ddagger}$ Technical University of Berlin, Str. d. 17.Juni 136, 10623 Berlin, Germany

$\S$ Technical University Darmstadt, Department of Mathematics, Dolivostr. 15, 64293 Darmstadt, Germany
} 
an $L^{2}$-misfit between the reconstruction pertinent to a certain number of experiments and the data usually collected in measurements devices placed outside or on the boundary of the observed region, plus a regularization term to obtain the appropriate regularity of the unknown function. The choice of the regularization is crucial for the quality of the reconstruction and for qualitative properties. Popular regularizers are the $L^{2}$-norm (Tikhonov), the $H^{1}$-norm or the total variation (TV) semi-norm. Recently, the latter has received a considerable amount of attention due to its capacity of preserving discontinuities while still sufficiently filtering noise.

Alternatively, based on a priori considerations, in this paper we take the approach of directly working in a class of functions with a given regularity and structural properties. In fact, we consider the case of piecewise constant functions

$$
\sigma=\sum_{i=0}^{n} \sigma_{i} \chi_{\Omega_{i}}
$$

with discontinuity sets $\partial \Omega_{i}$ having finite perimeter, i.e. a subclass of functions of bounded variation. Here, $\chi_{\Omega_{i}}$ denotes the indicator function of a set $\Omega_{i} \subset \mathbb{R}^{N},\left\{\sigma_{i}\right\}_{i=0}^{n} \in \mathbb{R}^{n}$ and $\left\{\Omega_{i}\right\}_{i=0}^{n}$ is a partitioning of the set $\Omega$. Such a structural assumption is often meaningful in applications, for instance in nondestructive testing, geophysics, or in medicine, where each organs have their respective electrical properties. Using the assumption of piecewise constant parameter functions, then the main issue is to localize the discontinuity sets of $\sigma$, i.e. to determine the partition $\left\{\Omega_{i}\right\}_{i=0}^{n}$. In this framework, the inverse problem takes the form of a shape optimization problem. Such geometry based approaches have been successfully applied for the detection of sharp interfaces in inverse problems and image analysis in the last few years; see for instance $[3,5,13,14,18,21-23,25,29,36]$

The rest of the paper is organized as follows. In Section 2 the geometry of the problem is discussed, and a shape optimization approach is formulated to solve the inverse problem. In Section 3 the forward problem of the eddy current model is studied. Moreover, its variational formulation is given and existence and regularity of the solution is proved. Higher regularity results are also obtained using additional regularity assumptions on the data. The notion of shape differentiability is defined in Section 4 and a saddle point formulation is used to compute the shape derivative. Further, in Sections 4.5 and 4.6, the domain and boundary expressions of the shape gradient are obtained, respectively. The paper ends by drawing conclusions.

2. Problem formulation. Let $\Omega \subset \mathbb{R}^{3}$ be a bounded and simply connected Lipschitz domain. We assume that inside this domain, there resides a Lipschitz domain $\Omega_{C}$ satisfying $\bar{\Omega}_{C} \subset \Omega$. This subdomain consists of conducting biological tissues. For simplicity, we assume that $\Omega_{C}$ has a connected boundary $\Gamma:=\partial \Omega_{C}$. Further, the set $\Omega_{I}:=\Omega \backslash \overline{\Omega_{C}}$ is assumed to be a non-conducting region; see Figure 2.1 for a description of the geometry. We consider an applied current density $\boldsymbol{J}$ generated by an open Lipschitz set of transmitter coils $\Omega_{T}$ which is strictly contained in $\Omega_{I}$, i.e., $\bar{\Omega}_{T} \subset \Omega_{I}$. Note that the applied current $\boldsymbol{J}$ is introduced in the non-conducting region $\Omega_{I}$. This describes the physical situation where the coils only induce a magnetic field and are not affected by the corresponding reaction field from the eddy current.

The $\boldsymbol{E}$-based time-harmonic eddy current equations are described by

$$
\left\{\begin{array}{rlrl}
\operatorname{curl} \mu^{-1} \operatorname{curl} \boldsymbol{E}+i \omega \sigma \boldsymbol{E} & =0 & & \text { in } \Omega_{C}, \\
\operatorname{curl} \mu^{-1} \operatorname{curl} \boldsymbol{E} & =-i \omega \boldsymbol{J} & & \text { in } \Omega_{I}, \\
\operatorname{div} \boldsymbol{E} & =0 & & \text { in } \Omega_{I}, \\
\mu^{-1} \operatorname{curl} \boldsymbol{E} \times \boldsymbol{n} & =0 & & \text { on } \partial \Omega, \\
\boldsymbol{E} \cdot \boldsymbol{n} & =0 & & \text { on } \partial \Omega, \\
\llbracket \boldsymbol{E} \times \boldsymbol{n} \rrbracket_{\Gamma}=\llbracket \mu^{-1} \operatorname{curl} \boldsymbol{E} \times \boldsymbol{n} \rrbracket_{\Gamma}=0 \text { on } \Gamma .
\end{array}\right.
$$




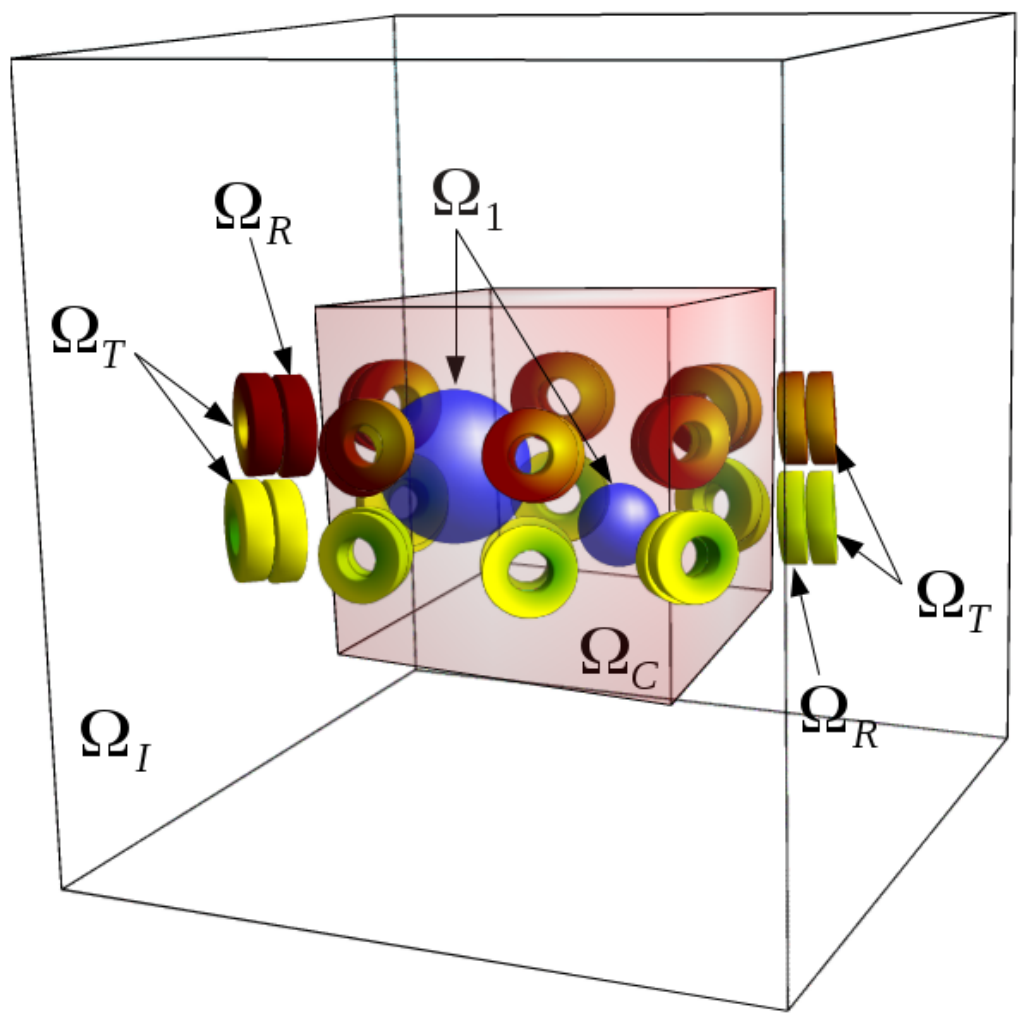

FIG. 2.1. Description of the geometry. The transmitter coils $\Omega_{T}$ and receiver coils $\Omega_{R}$ are placed on two annuli around the conducting region $\Omega_{C}$. The insulating region is a large box denoted by $\Omega_{I}$. The inclusions $\Omega_{1}$ are contained in $\Omega_{C}$.

Here, $\boldsymbol{E}$ denotes the electric field, $\omega$ the angular frequency, $i$ the imaginary unit, $\boldsymbol{n}$ the outward unit normal vector, and $\llbracket \cdot \rrbracket_{\Gamma}$ the jump of a quantity across the interface $\Gamma$. Further, the scalar function $\mu$ is the magnetic permeability of $\Omega$, and $\sigma$ denotes the electric conductivity of $\Omega_{C}$. In this setting, the magnetic permeability $\mu$ is assumed to be known data given by

$$
\mu= \begin{cases}\mu_{C} & \text { in } \Omega_{C}, \\ \mu_{I} & \text { in } \Omega_{I},\end{cases}
$$

with positive constants $\mu_{C}$ and $\mu_{I}$ representing the magnetic permeability of $\Omega_{C}$ and $\Omega_{I}$, respectively. Furthermore, the applied current density is assumed to satisfy:

$$
\boldsymbol{J} \equiv 0 \text { in } \Omega_{I} \backslash \Omega_{T}, \quad \operatorname{div} \boldsymbol{J} \equiv 0 \text { in } \Omega_{T}, \quad \boldsymbol{J} \cdot \boldsymbol{n} \equiv 0 \text { on } \partial \Omega_{T} .
$$

In order to model the receiver coils, we introduce an open set $\Omega_{R}$ satisfying $\overline{\Omega_{R}} \subset \Omega_{I}$ and $\overline{\Omega_{R}} \cap \overline{\Omega_{T}}=\emptyset$. 
This three-dimensional set $\Omega_{R}$ contains different receiver coils in the sense that

$$
\Omega_{R}=\bigcup_{k=1}^{n} R_{k}, \quad n \in \mathbb{N}
$$

where $R_{1}, \ldots, R_{n}$ are Lipschitz domains satisfying $\overline{R_{k}} \cap \overline{R_{j}}=\emptyset$ for all $k, j \in\{1, \ldots, n\}$. Following [34,38], the measurement in the $k$-th receiver coil $R_{k}$ is defined as

$$
\mathcal{M}^{k}(\boldsymbol{E})=\int_{R_{k}} \boldsymbol{E} \cdot \mathbf{J}_{k}
$$

where every $\mathbf{J}_{k}, k=1, \ldots, n$, is a given current density for an artificial unit current passing through the receiver coil $R_{k}$.

The inverse problem consists in reconstructing the conductivity $\sigma: \Omega_{C} \rightarrow \mathbb{R}$ from reference measurements $\mathcal{M}^{k, r e f}$ on $R_{k}$. For this purpose we introduce

$$
\mathfrak{J}(\boldsymbol{E}, \sigma):=K(\boldsymbol{E})+\kappa L(\sigma),
$$

where

$$
K(\boldsymbol{E}):=\frac{1}{2} \sum_{k=1}^{n}\left|\mathcal{M}^{k}(\boldsymbol{E})-\mathcal{M}^{k, r e f}\right|^{2} \quad \text { and } \quad L(\sigma)=\int_{\Omega_{C}}|D \sigma|,
$$

i.e., the functional $\mathfrak{J}$ is composed of a data misfit term $K$ and a total variation regularization term $L$. Here, $\kappa>0$ denotes a reguarization parameter, and as usual we have

$$
\int_{\Omega_{C}}|D \sigma|=\sup \left\{\int_{\Omega_{C}} \sigma \operatorname{div} \mathbf{p}: \mathbf{p} \in \mathbf{C}_{0}^{1}\left(\Omega_{C}\right),|\mathbf{p}(x)| \leq 1, \forall x \in \Omega_{C}\right\},
$$

where $\mathbf{C}_{0}^{1}\left(\Omega_{C}\right):=C_{0}^{1}\left(\Omega_{C}\right)^{3}$ is the space of continuously differentiable functions with compact support in $\Omega_{C}$. In what follows, given $\sigma$, let $\boldsymbol{E}(\sigma)$ denote the solution of (2.1). This allows us to introduce the reduced functional

$$
J(\sigma):=\mathfrak{J}(\boldsymbol{E}(\sigma), \sigma) .
$$

In many applications of MIT the unknown electric conductivity $\sigma$ of biological tissues $\Omega_{C}$ can be assumed piecewise constant, i.e.,

$$
\sigma=\sum_{j=0}^{n_{\sigma}} \sigma_{j} \chi_{\Omega_{j}}
$$

where $\left\{\Omega_{j}\right\}_{j=0}^{n_{\sigma}}$ is a partitioning of $\Omega_{C}, n_{\sigma} \in \mathbb{N}$ the number of inclusions, and $\chi_{\Omega_{j}}$ the characteristic function on the set $\Omega_{j}$. For every $j=1, \ldots, n_{\sigma}$, we assume that

$$
\Omega_{j} \in \mathcal{O}:=\left\{\mathcal{D} \subset \Omega_{C}: \mathcal{D} \text { is an open set of class } \mathcal{C}^{2,1} \text { and } \inf _{x \in \mathcal{D}, y \in \partial \Omega_{C}}|x-y| \geq d_{0}\right\}
$$

with a given positive constant $d_{0}$. Due to the last condition in $\mathcal{O}$, the boundary $\Gamma_{j}:=\partial \Omega_{j}$ for every $j=1, \ldots, n_{\sigma}$ does not touch the boundary of $\Omega_{C}$. Thus, the regularization term becomes

$$
\int_{\Omega}|D \sigma|=\sum_{j=1}^{n_{\sigma}}\left|\sigma_{0}-\sigma_{j}\right| \mathcal{P}\left(\Gamma_{j}\right)
$$


where $\mathcal{P}\left(\Gamma_{j}\right)$ stands for the perimeter of $\Gamma_{j}$. Note that the unknown conductivity $\sigma$ may be written as a mapping

$$
\sigma:\left(\mathbb{R}^{+}\right)^{n_{\sigma}+1} \times \mathcal{O}^{n_{\sigma}+1} \rightarrow \mathrm{BV}\left(\Omega_{C}\right), \quad\left(\left\{\sigma_{j}\right\}_{j=0}^{n_{\sigma}},\left\{\Omega_{j}\right\}_{j=0}^{n_{\sigma}}\right) \mapsto \sigma\left(\left\{\sigma_{j}\right\}_{j=0}^{n_{\sigma}},\left\{\Omega_{j}\right\}_{j=0}^{n_{\sigma}}\right),
$$

where $B V\left(\Omega_{C}\right)$ is the Banach space of functions of bounded variation on $\Omega_{C}$; see [16] for a general definition. This gives rise to the so-called shape functional

$$
\begin{aligned}
\mathcal{J}\left(\left\{\sigma_{j}\right\}_{j=0}^{n_{\sigma}},\left\{\Omega_{j}\right\}_{j=0}^{n_{\sigma}}\right): & =J\left(\sigma\left(\left\{\sigma_{j}\right\}_{j=0}^{n_{\sigma}},\left\{\Omega_{j}\right\}_{j=0}^{n_{\sigma}}\right)\right) \\
& =\frac{1}{2} \sum_{k=1}^{n}\left|\mathcal{M}^{k}\left(\boldsymbol{E}\left(\sigma\left(\left\{\sigma_{j}\right\}_{j=0}^{n_{\sigma}},\left\{\Omega_{j}\right\}_{j=0}^{n_{\sigma}}\right)\right)\right)-\mathcal{M}^{k, r e f}\right|^{2}+\kappa \sum_{i=1}^{n_{\sigma}}\left|\sigma_{0}-\sigma_{i}\right| \mathcal{P}\left(\Gamma_{i}\right) .
\end{aligned}
$$

In view of (2.9), the functional $\mathcal{J}$ does not explicitly depend on $\Omega_{0}$. Therefore, the inverse problem reads as follows:

$$
\min _{\left\{\sigma_{j}\right\}_{j=0}^{n_{\sigma}},\left\{\Omega_{j}\right\}_{j=1}^{n_{\sigma}}} \mathcal{J}\left(\left\{\sigma_{j}\right\}_{j=0}^{n_{\sigma}},\left\{\Omega_{j}\right\}_{j=1}^{n_{\sigma}}\right)
$$

where $\left\{\sigma_{j}\right\}_{j=0}^{n_{\sigma}} \in\left(\mathbb{R}^{+}\right)^{n_{\sigma}+1}$ and $\Omega_{j} \in \mathcal{O}$ for all $j=1, \ldots, n_{\sigma}$. In this paper, we only consider the case where $n_{\sigma}=1$ and $\sigma_{0}, \sigma_{1}$ are fixed given constant (see Sect. 3). The analysis can be straightforwardly extended to the general case $n_{\sigma}>1$.

3. Forward problem. We start by introducing notations and function spaces. The notation $\|\cdot\|_{X}$ is used for a standard norm in a Banach space $X$. If $X$ is continuously embedded into another Banach space $Y$, then we write $X \hookrightarrow Y$. We use a bold typeface to indicate a three-dimensional vector function or a Banach space of three-dimensional vector functions. For any Lipschitz domain $\mathcal{D} \subset \mathbb{R}^{3}$, let us define

$$
\begin{gathered}
\boldsymbol{H}(\operatorname{curl} ; \mathcal{D}):=\left\{\boldsymbol{v} \in \boldsymbol{L}^{2}(\mathcal{D}) \mid \operatorname{curl} \boldsymbol{v} \in \boldsymbol{L}^{2}(\mathcal{D})\right\}, \\
\boldsymbol{H}(\operatorname{div} ; \mathcal{D}):=\left\{\boldsymbol{v} \in \boldsymbol{L}^{2}(\mathcal{D}) \mid \operatorname{div} \boldsymbol{v} \in L^{2}(\mathcal{D})\right\},
\end{gathered}
$$

where the curl- and div-operators are understood in the distributional sense. The space $\boldsymbol{L}^{2}(\mathcal{D})$ denotes the Hilbert space of square Lebesgue integrable complex-valued three-dimensional vector functions on $\mathcal{D}$. Also, we denote by $\boldsymbol{H}^{s}(\mathcal{D})$, with $s \in \mathbb{R}^{+}$, a standard Sobolev space [1]. Let us now introduce the state space

$$
\boldsymbol{V}:=\left\{\boldsymbol{v} \in \boldsymbol{H}(\operatorname{curl} ; \Omega) \cap \boldsymbol{H}\left(\operatorname{div} ; \Omega_{I}\right) \mid \boldsymbol{v} \cdot \boldsymbol{n}=0 \text { on } \partial \Omega\right\},
$$

where the normal trace $\boldsymbol{v} \cdot \boldsymbol{n}$ is understood in the distributional sense (see [15]). The space $\boldsymbol{V}$ is endowed with the following norm:

$$
\|\boldsymbol{v}\|_{\boldsymbol{V}}:=\left(\|\boldsymbol{v}\|_{\boldsymbol{L}^{2}(\Omega)}^{2}+\|\operatorname{curl} \boldsymbol{v}\|_{\boldsymbol{L}^{2}(\Omega)}^{2}+\|\operatorname{div} \boldsymbol{v}\|_{L^{2}\left(\Omega_{I}\right)}^{2}\right)^{\frac{1}{2}}
$$

3.1. Variational formulation. The weak formulation of (2.1) reads: Given $\boldsymbol{J} \in \boldsymbol{L}^{2}\left(\Omega_{I}\right)$, find $\boldsymbol{E} \in \boldsymbol{V}$ such that

$$
\int_{\Omega} \mu^{-1} \operatorname{curl} \boldsymbol{E} \cdot \operatorname{curl} \overline{\boldsymbol{v}}+i \omega \int_{\Omega_{C}} \sigma \boldsymbol{E} \cdot \overline{\boldsymbol{v}}+\int_{\Omega_{I}} \operatorname{div} \boldsymbol{E} \operatorname{div} \overline{\boldsymbol{v}}=-i \omega \int_{\Omega_{T}} \boldsymbol{J} \cdot \overline{\boldsymbol{v}} \quad \forall \boldsymbol{v} \in \boldsymbol{V}
$$


where $\overline{\boldsymbol{v}}$ denotes the complex conjugate of $\boldsymbol{v}$. Existence of a unique solution to (3.1) is a well-known result which follows from the Lax-Milgram lemma (see [2]). We shall show that (3.1) admits a unique solution $\boldsymbol{E} \in \boldsymbol{V}$ satisfying $\operatorname{div} \boldsymbol{E}=0$ in $\Omega_{I}$. Thus, (3.1) is equivalent to the standard variational formulation of (2.1). The key point for the existence result is the upcoming Poincaré-Friedrichs-type inequality, which holds in our case due to our simplifying geometric assumption that both $\Omega_{C}$ and $\Omega$ are simply connected with connected boundaries.

LEMma 3.1 ( [2, Lemma 2.2]). There exists a constant $c>0$, depending only on $\Omega$, such that

$$
\|\boldsymbol{v}\|_{\boldsymbol{L}^{2}\left(\Omega_{I}\right)} \leq c\left(\|\operatorname{curl} \boldsymbol{v}\|_{\boldsymbol{L}^{2}(\Omega)}+\|\boldsymbol{v}\|_{\boldsymbol{L}^{2}\left(\Omega_{C}\right)}+\|\operatorname{div} \boldsymbol{v}\|_{L^{2}\left(\Omega_{I}\right)}\right) \quad \forall \boldsymbol{v} \in \boldsymbol{V} .
$$

Based on Lemma 3.1, we easily obtain the following existence and uniqueness result:

LEMmA 3.2. Assume that $\sigma: \Omega_{C} \rightarrow \mathbb{R}$ satisfies $\underline{\sigma} \leq \sigma \leq \bar{\sigma}$ almost everywhere (a.e.) in $\Omega_{C}$ for some positive real numbers $0<\underline{\sigma}<\bar{\sigma}$. Then, the weak formulation (3.1) admits a unique solution $\boldsymbol{E}=\boldsymbol{E}(\sigma) \in \boldsymbol{V}$ satisfying $\operatorname{div} \boldsymbol{E}=0$ in $\Omega_{I}$.

Proof. We introduce the sesquilinear form $\boldsymbol{a}_{\sigma}: \boldsymbol{V} \times \boldsymbol{V} \rightarrow \mathbb{C}$, defined by

$$
\boldsymbol{a}_{\sigma}(\boldsymbol{u}, \boldsymbol{v}):=\int_{\Omega} \mu^{-1} \operatorname{curl} \boldsymbol{u} \cdot \operatorname{curl} \overline{\boldsymbol{v}}+i \omega \int_{\Omega_{C}} \sigma \boldsymbol{u} \cdot \overline{\boldsymbol{v}}+\int_{\Omega_{I}} \operatorname{div} \boldsymbol{u} \operatorname{div} \overline{\boldsymbol{v}} .
$$

Since the scalar functions $\mu: \Omega \rightarrow \mathbb{R}$ and $\sigma: \Omega_{C} \rightarrow \mathbb{R}$ are bounded, the sesquilinear form $\boldsymbol{a}_{\sigma}: \boldsymbol{V} \times \boldsymbol{V} \rightarrow \mathbb{C}$ is bounded. Further, by our assumption, we have

$$
\frac{1}{\sqrt{2}}\left(\max \left\{\mu_{C}, \mu_{I}\right\}^{-1}\|\operatorname{curl} \boldsymbol{v}\|_{\boldsymbol{L}^{2}(\Omega)}^{2}+\underline{\sigma} \omega\|\boldsymbol{v}\|_{\boldsymbol{L}^{2}\left(\Omega_{C}\right)}^{2}+\|\operatorname{div} \boldsymbol{v}\|_{L^{2}\left(\Omega_{I}\right)}^{2}\right) \leq\left|\boldsymbol{a}_{\sigma}(\boldsymbol{v}, \boldsymbol{v})\right| \quad \forall \boldsymbol{v} \in \boldsymbol{V} .
$$

Therefore, in view of Lemma 3.1, the sesquilinear form $\boldsymbol{a}_{\sigma}: \boldsymbol{V} \times \boldsymbol{V} \rightarrow \mathbb{C}$ is coercive. Moreover, the right hand side in (3.1) induces a continuous linear functional on $\boldsymbol{V}$. Thus, the Lax-Milgram lemma implies that the weak formulation (3.1) admits a unique solution $\boldsymbol{E}=\boldsymbol{E}(\sigma) \in \boldsymbol{V}$.

To show that $\operatorname{div} \boldsymbol{E}=0$ in $\Omega_{I}$, let us define

$$
\mathcal{D}(\Delta):=\left\{\psi \in H^{1}\left(\Omega_{I}\right) \mid \nabla \psi \in \boldsymbol{H}\left(\operatorname{div} ; \Omega_{I}\right), \quad \psi_{\mid \Gamma}=0, \quad \nabla \psi \cdot \boldsymbol{n}=0 \text { on } \partial \Omega\right\} .
$$

By definition, we see that every gradient field $\boldsymbol{v}=\nabla \hat{\psi}$ with

$$
\hat{\psi}= \begin{cases}\psi \in \mathcal{D}(\Delta) & \text { in } \Omega_{I}, \\ 0 & \text { in } \Omega_{C}\end{cases}
$$

belongs to $\boldsymbol{V}$, since $\psi$ satisfies $\psi_{\mid \Gamma}=0$ and $\nabla \psi \cdot \boldsymbol{n}=0$ on $\partial \Omega$. Then, setting $\boldsymbol{v}=\nabla \hat{\psi}$ in (3.1) and using (2.3) yields

$$
\int_{\Omega_{I}}(\operatorname{div} \boldsymbol{E})(\operatorname{div}(\overline{\nabla \psi}))=0 \quad \forall \psi \in \mathcal{D}(\Delta),
$$

where we have also used the identity $\operatorname{curl} \nabla \equiv 0$. Consider now the following variational problem

$$
(\nabla \phi, \nabla \psi)_{L^{2}\left(\Omega_{I}\right)}=-(u, \psi)_{L^{2}\left(\Omega_{I}\right)} \quad \forall \psi \in H_{\Gamma}^{1}\left(\Omega_{I}\right):=\left\{\psi \in H^{1}\left(\Omega_{I}\right) \mid \psi_{\mid \Gamma}=0\right\} .
$$

For every $u \in L^{2}\left(\Omega_{I}\right)$, the Lax-Milgram-Lemma implies that (3.5) admits a unique solution $\phi \in H_{\Gamma}^{1}\left(\Omega_{I}\right)$. Further, in view of the distributional definition of the divergence and the normal trace, this solution satisfies

$$
\operatorname{div}(\nabla \phi)=u \quad \text { and } \quad \nabla \phi \cdot \boldsymbol{n}=0 \text { on } \partial \Omega,
$$


and so $\phi \in \mathcal{D}(\Delta)$. Consequently, we obtain from (3.5) that

$$
\int_{\Omega_{I}}(\operatorname{div} \boldsymbol{E}) \bar{u}=0 \quad \forall u \in L^{2}\left(\Omega_{I}\right)
$$

yielding $\operatorname{div} \boldsymbol{E}=0$ in $\Omega_{I}$ which concudes the proof.

3.2. Higher regularity. In the previous section we have shown the existence of a solution $\boldsymbol{E} \in \boldsymbol{V}$ to (3.1). For obtaining the boundary representation of the shape derivative in Section 4.6, we need higher regularity of $\boldsymbol{E}$, which is the purpose of this section.

Lemma 3.3. Let $\sigma: \Omega_{C} \rightarrow \mathbb{R}$ be bounded and uniformly positive. Let $\boldsymbol{E}=\boldsymbol{E}(\sigma) \in \boldsymbol{V}$ be the solution of the weak formulation (3.1). Then, the regularity property

$$
\operatorname{curl} \boldsymbol{E} \in \boldsymbol{H}^{1}(\mathcal{D})
$$

holds true for every open set $\mathcal{D}$ satisfying $\overline{\mathcal{D}} \subset \Omega_{C}$.

Proof. Inserting $\boldsymbol{v}=\hat{\boldsymbol{\psi}}$ in (3.1) with

$$
\hat{\boldsymbol{\psi}}= \begin{cases}\boldsymbol{\psi} \in \mathcal{C}_{0}^{\infty}\left(\Omega_{C}\right)^{3} & \text { in } \Omega_{C}, \\ 0 & \text { otherwise }\end{cases}
$$

results in

$$
\int_{\Omega_{C}} \mu^{-1} \operatorname{curl} \boldsymbol{E} \cdot \operatorname{curl} \overline{\boldsymbol{\psi}}+i \omega \int_{\Omega_{C}} \sigma \boldsymbol{E} \cdot \overline{\boldsymbol{\psi}}=0 \quad \forall \boldsymbol{\psi} \in \mathcal{C}_{0}^{\infty}\left(\Omega_{C}\right)^{3}
$$

and hence, by (2.2), we obtain that

$$
\int_{\Omega_{C}} \operatorname{curl} \boldsymbol{E} \cdot \operatorname{curl} \overline{\boldsymbol{\psi}}=\int_{\Omega_{C}}\left(-i \omega \mu_{C} \sigma \boldsymbol{E}\right) \cdot \overline{\boldsymbol{\psi}} \quad \forall \boldsymbol{\psi} \in \mathcal{C}_{0}^{\infty}\left(\Omega_{C}\right)^{3} .
$$

For this reason, the distributional definition of the curl-operator yields

$$
\operatorname{curl} \operatorname{curl} \boldsymbol{E}=-i \omega \mu_{C} \sigma \boldsymbol{E} \in \boldsymbol{L}^{2}\left(\Omega_{C}\right) \quad \Longrightarrow \quad \operatorname{curl} \boldsymbol{E} \in \boldsymbol{H}\left(\operatorname{curl} ; \Omega_{C}\right)
$$

As $\Gamma$ is connected, a well-known result (see [28, Theorem 3.38]) yields the existence of a vector potential $\boldsymbol{A} \in \boldsymbol{H}^{1}\left(\Omega_{C}\right)$ satisfying $\operatorname{div} \boldsymbol{A}=0$ in $\Omega_{C}$ such that

$$
\operatorname{curl} E=\operatorname{curl} A \text { in } \Omega_{C} .
$$

In view of (3.6), it follows that

$$
-i \omega \mu_{C} \sigma \boldsymbol{E}=\operatorname{curl} \operatorname{curl} \boldsymbol{E}=\operatorname{curl} \operatorname{curl} \boldsymbol{A} \underbrace{=}_{\operatorname{div} \boldsymbol{A}=0} \boldsymbol{\Delta} \boldsymbol{A} \text { in } \Omega_{C},
$$

from which we deduce that

$$
\Delta A_{j} \in L^{2}\left(\Omega_{C}\right) \text { for } j=1,2,3 .
$$

For this reason, a well-known interior elliptic regularity result implies that

$$
A_{j} \in H^{2}(\mathcal{D}) \text { for } j=1,2,3,
$$


holds true for every open set $\mathcal{D}$ satisfying $\overline{\mathcal{D}} \subset \Omega_{C}$. In conclusion, the assertion follows from the above regularity and (3.7).

Based on Lemma 3.3, we are now able to prove an interior regularity result for the solution $\boldsymbol{E}$ of (3.1). The following theorem will be important for our subsequent analysis.

THEOREM 3.4. Let $\Omega_{1}$ be an open $\mathcal{C}^{2,1}$-set satisfying $\bar{\Omega}_{1} \subset \Omega_{C}$. We set $\Omega_{0}:=\Omega_{C} \backslash \bar{\Omega}_{1}$ and

$$
\sigma=\sigma_{0} \chi_{\Omega_{0}}+\sigma_{1} \chi_{\Omega_{1}} \quad \sigma_{0}, \sigma_{1} \in \mathbb{R}^{+} .
$$

Then, the solution $\boldsymbol{E}=\boldsymbol{E}(\sigma) \in \boldsymbol{V}$ of the weak formulation (3.1) satisfies

$$
\boldsymbol{E} \in \boldsymbol{H}^{2}\left(\Omega_{1}\right) .
$$

Further, for every open set $\mathcal{D}$ of class $\mathcal{C}^{2,1}$ satisfying $\bar{\Omega}_{1} \subset \mathcal{D}$ and $\overline{\mathcal{D}} \subset \Omega_{C}$, we have

$$
\boldsymbol{E} \in \boldsymbol{H}^{2}\left(\mathcal{D} \backslash \bar{\Omega}_{1}\right)
$$

Proof. Let $\mathcal{D}$ be an open $\mathcal{C}^{2,1}$-set satisfying $\bar{\Omega}_{1} \subset \mathcal{D}$ and $\overline{\mathcal{D}} \subset \Omega_{C}$. We find a $\mathcal{C}^{\infty}$-domain $\mathfrak{D}$ with a connected boundary satisfying $\overline{\mathcal{D}} \subset \mathfrak{D} \subset \Omega_{C}$. Setting $\boldsymbol{v}=\nabla \hat{\phi}$ in (3.1) with

$$
\hat{\phi}= \begin{cases}\phi \in H_{0}^{1}(\mathfrak{D}) & \text { in } \mathfrak{D}, \\ 0 & \text { otherwise, }\end{cases}
$$

yields

$$
\int_{\mathfrak{D}} \sigma \boldsymbol{E} \cdot \nabla \bar{\phi}=0 \quad \forall \phi \in H_{0}^{1}(\mathfrak{D}) .
$$

Furthermore, applying the Helmholtz decomposition [28, Theorem 3.45], the vector field $\boldsymbol{E}$ considered as a function in $\boldsymbol{L}^{2}(\mathfrak{D})$ can be decomposed as

$$
\boldsymbol{E}=\nabla p+\operatorname{curl} \boldsymbol{A} \text { in } \mathfrak{D},
$$

with $p \in H_{0}^{1}(\mathfrak{D})$ and $\boldsymbol{A} \in \boldsymbol{H}^{1}(\mathfrak{D})$ satisfying $\operatorname{div} \boldsymbol{A}=0$ in $\mathfrak{D}$. By Lemma 3.3, it follows that

$$
\boldsymbol{H}^{1}(\mathfrak{D}) \ni \operatorname{curl} \boldsymbol{E}=\operatorname{curl} \operatorname{curl} \boldsymbol{A}=\boldsymbol{\Delta A},
$$

and hence, by classical results on interior elliptic regularity, we infer that

$$
\boldsymbol{A} \in \boldsymbol{H}^{3}(\mathcal{D}) \quad \Longrightarrow \quad \operatorname{curl} \boldsymbol{A} \in \boldsymbol{H}^{2}(\mathcal{D}) .
$$

In particular, as $\bar{\Omega}_{1} \subset \mathcal{D}$, we obtain $\operatorname{curl} \boldsymbol{A} \in \boldsymbol{H}^{2}\left(\Omega_{1}\right)$. Denote by $\boldsymbol{n}_{1}$ the outer unit normal vector to $\Omega_{1}$. Since $\Omega_{1}$ is of class $\mathcal{C}^{2,1}$, we have $\boldsymbol{n}_{1} \in \mathcal{C}^{1,1}\left(\Gamma_{1}\right)$, where $\Gamma_{1}:=\partial \Omega_{1}$. Thus, since curl $\boldsymbol{A} \in \boldsymbol{H}^{\frac{3}{2}}\left(\Gamma_{1}\right)$, we obtain

$$
\operatorname{curl} \boldsymbol{A} \cdot \boldsymbol{n}_{1} \in H^{\frac{3}{2}}\left(\Gamma_{1}\right) \text {. }
$$

Multiplying (3.10) by $\sigma \nabla \bar{\phi}$, where $\phi \in H_{0}^{1}(\mathfrak{D})$, and then integrating the resulting equality over $\mathfrak{D}$ yields

$$
\int_{\mathfrak{D}} \sigma \boldsymbol{E} \cdot \nabla \bar{\phi}=\int_{\mathfrak{D}} \sigma \nabla p \cdot \nabla \bar{\phi}+\int_{\mathfrak{D}} \sigma \operatorname{curl} \boldsymbol{A} \cdot \nabla \bar{\phi} \quad \forall \phi \in H_{0}^{1}(\mathfrak{D}) .
$$


Then, in view of (3.8) and (3.9), it follows that

$$
\begin{aligned}
\int_{\mathfrak{D}} \sigma \nabla p \cdot \nabla \bar{\phi} & =-\sigma_{0} \int_{\mathfrak{D} \backslash \Omega_{1}} \operatorname{curl} \boldsymbol{A} \cdot \nabla \bar{\phi}-\sigma_{1} \int_{\Omega_{1}} \operatorname{curl} \boldsymbol{A} \cdot \nabla \bar{\phi} \\
& =\left(\sigma_{0}-\sigma_{1}\right) \int_{\Gamma_{1}} \operatorname{curl} \boldsymbol{A} \cdot \boldsymbol{n}_{1} \bar{\phi}, \quad \forall \phi \in H_{0}^{1}(\mathfrak{D}),
\end{aligned}
$$

where we have also used Green's theorem as well as the identity div curl $\equiv 0$ for the last equality. Thanks to (3.12), a well-known regularity result for elliptic transmission problems (see [7, Chapter 5, Theorem 5.2.1.]) implies that the solution $p \in H_{0}^{1}(\mathfrak{D})$ of (3.13) satisfies

$$
p \in H^{3}\left(\Omega_{1}\right) \quad \text { and } \quad p \in H^{3}\left(\mathfrak{D} \backslash \bar{\Omega}_{1}\right) .
$$

From (3.11) and (3.14), we finally come to the conclusion that

$$
\boldsymbol{E}=\nabla p+\operatorname{curl} \boldsymbol{A} \in \boldsymbol{H}^{2}\left(\Omega_{1}\right) \quad \text { and } \quad \boldsymbol{E}=\nabla p+\operatorname{curl} \boldsymbol{A} \in \boldsymbol{H}^{2}\left(\mathcal{D} \backslash \bar{\Omega}_{1}\right) .
$$

This completes the proof.

4. Shape sensitivity analysis. The major difficulty in dealing with sets of shapes is that they do not have a vector space structure. In order to be able to define shape derivatives and study the sensitivity of shape functionals, we need to construct such a structure for the shape spaces. In the literature, this is done by considering perturbations of an initial domain; see [9, 19,33].

Therefore, essentially two types of domain perturbations are considered in general. The first one is the method of perturbation of the identity operator, and the second one is the velocity or speed method which is based on the deformation obtained by the flow induced by a velocity field. The speed method is more general than the method of perturbation of the identity operator. The equivalence between deformations obtained by a family of transformations and deformations obtained by the flow of velocity field may be shown $[9,33]$. The method of perturbation of the identity operator is a particular kind of domain transformation, and in this paper the main results will be given using a simplified speed method. We point out that using one or the other is rather a matter of preference as several classical textbooks and authors rely on the method of perturbation of the identity operator as well.

For the presentation of the speed method, we mainly rely on $[9,33]$. We also restrict ourselves to shape perturbations by autonomous vector fields, i.e., time-independent vector fields. Let $\boldsymbol{\theta}: \mathbb{R}^{3} \rightarrow \mathbb{R}^{3}$ be an autonomous vector field and assume that

$$
\boldsymbol{\theta} \in \boldsymbol{\Theta}^{k}:=\left\{\boldsymbol{\theta} \in \mathcal{C}^{k}\left(\mathbb{R}^{3}, \mathbb{R}^{3}\right): \boldsymbol{\theta} \text { has compact support }\right\},
$$

with $k \geq 0$. For $\tau>0$, we introduce a family of transformations

$$
T_{t}(\boldsymbol{\theta})(X)=x(t, X)
$$

as the solution to the ordinary differential equation

$$
\left\{\begin{aligned}
\frac{d}{d t} x(t, X) & =\boldsymbol{\theta}(x(t, X)), \quad 0<t<\tau, \\
x(0, X) & =X \in \mathbb{R}^{3} .
\end{aligned}\right.
$$

For sufficiently small $\tau$ the system (4.3) has a unique solution (see e.g. [33]). Now, the transformation $T_{t}(\boldsymbol{\theta})$ allows to define a family of domains

$$
\Omega(t):=T_{t}(\boldsymbol{\theta})(\Omega),
$$


which we will use for the differentiation of the shape functional. We refer to [9, Chapter 7] and [33, Theorem 2.16] for theorems establishing the regularity of the transformation $T_{t}(\boldsymbol{\theta})$.

Let $J(\Omega)$ be a shape functional well-defined for any measurable set $\Omega \subset \mathbb{R}^{3}$. We recall the following notions of differentiability with respect to the shape:

DEFINITION 4.1 (Eulerian semiderivative). Let $\boldsymbol{\theta} \in \boldsymbol{\Theta}^{k}$ with $k \geq 0$. Then the Eulerian semiderivative of the shape functional $J(\Omega)$ at $\Omega$ in the direction $\boldsymbol{\theta}$ is defined as the limit

$$
d J(\Omega ; \boldsymbol{\theta})=\lim _{t \searrow 0} \frac{J(\Omega(t))-J(\Omega)}{t},
$$

if the limit exists in $\mathbb{R}$.

DEFINITION 4.2 (Shape Differentiability). The functional $J(\Omega)$ is shape differentiable (or differentiable for simplicity) at $\Omega$ if it has a Eulerian semiderivative at $\Omega$ in all directions $\boldsymbol{\theta}$ and the map

$$
\boldsymbol{\theta} \mapsto d J(\Omega, \boldsymbol{\theta})
$$

is linear and continuous from $\Theta^{k}$ into $\mathbb{R}$. The map (4.6) is then sometimes denoted by $\nabla J(\Omega)$ and referred to as the shape gradient of $J$, and we have

$$
d J(\Omega, \boldsymbol{\theta})=\langle\nabla J(\Omega), \boldsymbol{\theta}\rangle_{\boldsymbol{\Theta}^{-k}, \boldsymbol{\Theta}^{k}},
$$

where $\Theta^{-k}$ is the dual of $\Theta^{k}$.

If the data are smooth enough, i.e. when the boundary of the domain $\Omega$ and the velocity field $\boldsymbol{\theta}$ are smooth enough (this will be observed later on), then the shape derivative has a particular structure. More precisely, it is concentrated on the boundary $\partial \Omega$ and depends only on the normal component of the velocity field $\boldsymbol{\theta}$ on the boundary $\partial \Omega$. This result, often called the structure theorem or Hadamard formula, is fundamental in shape optimization.

4.1. Inverse problem. We assume that the electric conductivity $\sigma: \Omega_{C} \rightarrow \mathbb{R}$ is a piecewise constant function. For the sake of simplicity of the presentation, we take $n_{\sigma}=1$, but the results for arbitrary $n_{\sigma}$ can be deduced straightforwardly. In this framework, $\sigma$ takes the following form:

$$
\sigma=\sigma_{0} \chi_{\Omega_{0}}+\sigma_{1} \chi_{\Omega_{1}},
$$

where $\Omega_{0}$ and $\Omega_{1}$ constitute a partition of $\Omega_{C}$, i.e., $\Omega_{0} \cap \Omega_{1}=\emptyset$ and $\Omega_{C}=\Omega_{0} \cup \Omega_{1}$ with

$$
\Omega_{1} \in \mathcal{O}=\left\{\mathcal{D} \subset \Omega_{C}: \mathcal{D} \text { is an open set of class } \mathcal{C}^{2,1} \text { and } \inf _{x \in \mathcal{D}, y \in \partial \Omega_{C}}|x-y| \geq d_{0}>0\right\} .
$$

We assume that $\sigma_{0}$ and $\sigma_{1}$ are fixed given positive constant. Therefore, due to the last condition in $\mathcal{O}$, the shape functional $\mathcal{J}\left(\left\{\sigma_{0}, \sigma_{1}\right\},\left\{\Omega_{0}, \Omega_{1}\right\}\right)$ depends only on the variable $\Omega_{1}$. Also, in order to emphasize the dependence of the function $\sigma$ on $\Omega_{1}$, we write

$$
\sigma\left(\Omega_{1}\right)=\sigma_{0} \chi_{\Omega_{0}}+\sigma_{1} \chi_{\Omega_{1}} .
$$

Now, the considered shape optimization problem reads as follows:

$$
\begin{aligned}
& \text { minimize } \mathcal{J}\left(\Omega_{1}\right):=\frac{1}{2} \sum_{k=1}^{n}\left|\mathcal{M}^{k}\left(\boldsymbol{E}\left(\sigma\left(\Omega_{1}\right)\right)\right)-\mathcal{M}^{k, \text { ref }}\right|^{2}+\kappa\left|\sigma_{0}-\sigma_{1}\right| \mathcal{P}\left(\Gamma_{1}\right), \\
& \text { subject to } \Omega_{1} \in \mathcal{O},
\end{aligned}
$$

where $\Gamma_{1}=\partial \Omega_{1}$, and $\boldsymbol{E}\left(\sigma\left(\Omega_{1}\right)\right) \in \boldsymbol{V}$ denotes the unique solution of (3.1) associated with the electric conductivity $\sigma=\sigma\left(\Omega_{1}\right)$. 
4.2. Saddle point formulation. The electric field $\boldsymbol{E}(\sigma)=\boldsymbol{E} \in \boldsymbol{V}$ may be split into its real and imaginary parts yielding

$$
\boldsymbol{E}=\boldsymbol{E}_{1}+i \boldsymbol{E}_{2}
$$

where $\boldsymbol{E}_{1}:=\mathcal{R} e \boldsymbol{E}$ and $\boldsymbol{E}_{2}:=\mathcal{I} m \boldsymbol{E}$. The same notation is also used for $\boldsymbol{J}$. In view of (4.10), the variational formulation (3.1) is equivalent to the following coupled formulation involving only real-valued functions:

$$
\begin{aligned}
& \left(\mu^{-1} \operatorname{curl} \boldsymbol{E}_{1}, \operatorname{curl} \boldsymbol{v}\right)_{\boldsymbol{L}^{2}(\Omega)}-\omega\left(\sigma \boldsymbol{E}_{2}, \boldsymbol{v}\right)_{\boldsymbol{L}^{2}\left(\Omega_{C}\right)}+\left(\operatorname{div} \boldsymbol{E}_{1}, \operatorname{div} \boldsymbol{v}\right)_{L^{2}\left(\Omega_{I}\right)}=\left(\omega \boldsymbol{J}_{2}, \boldsymbol{v}\right)_{\boldsymbol{L}^{2}\left(\Omega_{T}\right)} \\
& \left(\mu^{-1} \operatorname{curl} \boldsymbol{E}_{2}, \operatorname{curl} \boldsymbol{v}\right)_{\boldsymbol{L}^{2}(\Omega)}+\omega\left(\sigma \boldsymbol{E}_{1}, \boldsymbol{v}\right)_{\boldsymbol{L}^{2}\left(\Omega_{C}\right)}+\left(\operatorname{div} \boldsymbol{E}_{2}, \operatorname{div} \boldsymbol{v}\right)_{L^{2}\left(\Omega_{I}\right)}=-\left(\omega \boldsymbol{J}_{1}, \boldsymbol{v}\right)_{\boldsymbol{L}^{2}\left(\Omega_{T}\right)}
\end{aligned}
$$

for real-valued test functions $\boldsymbol{v}$. The coupled system (3.1) may actually be obtained from the real part or from the imaginary part of (2.1). Therefore it is equivalent to consider the variational formulation (3.1) or only the real or the imaginary part of it. This consideration allows to introduce now a real-valued Lagrangian where only the real part of (2.1) is penalized, but which allows to recover the complete state equations (4.11).

Following the theory developed in [9, Chapter 9, section 5], we build an appropriate Lagrangian functional $\mathscr{L}: \boldsymbol{V} \times \boldsymbol{V} \times \mathcal{O} \rightarrow \mathbb{R}$ to derive optimality conditions for the shape optimization problem (4.9):

$$
\begin{aligned}
\mathscr{L}\left(\boldsymbol{E}, \boldsymbol{F}, \Omega_{1}\right):=K(\boldsymbol{E}) & +\mathcal{R} e\left(\int_{\Omega} \mu^{-1} \operatorname{curl} \boldsymbol{E} \cdot \operatorname{curl} \overline{\boldsymbol{F}}+i \omega \int_{\Omega_{C}} \sigma\left(\Omega_{1}\right) \boldsymbol{E} \cdot \overline{\boldsymbol{F}}\right) \\
& +\mathcal{R} e\left(\int_{\Omega_{E}} \operatorname{div} \boldsymbol{E} \operatorname{div} \overline{\boldsymbol{F}}+i \omega \int_{\Omega_{T}} \boldsymbol{J} \cdot \overline{\boldsymbol{F}}\right) .
\end{aligned}
$$

Here, $\boldsymbol{F}$ is the dual variable. Since

$$
\sup _{\boldsymbol{F} \in \boldsymbol{V}} \mathscr{L}\left(\boldsymbol{E}, \boldsymbol{F}, \Omega_{1}\right)= \begin{cases}K\left(\boldsymbol{E}\left(\sigma\left(\Omega_{1}\right)\right)\right) & \text { if } \boldsymbol{E}=\boldsymbol{E}\left(\sigma\left(\Omega_{1}\right)\right) \\ +\infty & \text { if } \boldsymbol{E} \neq \boldsymbol{E}\left(\sigma\left(\Omega_{1}\right)\right)\end{cases}
$$

the objective functional $\mathcal{J}$ can be expressed through the following saddle point formulation:

$$
\mathcal{J}\left(\Omega_{1}\right)=\left(\inf _{\boldsymbol{E} \in \boldsymbol{V}} \sup _{\boldsymbol{F} \in \boldsymbol{V}} \mathscr{L}\left(\boldsymbol{E}, \boldsymbol{F}, \Omega_{1}\right)\right)+\kappa L\left(\sigma\left(\Omega_{1}\right)\right) .
$$

The Lagrangian $\mathscr{L}$ is convex and continuous with respect to $\boldsymbol{E}$. Moreover, it is linear and continuous, and thus concave, with respect to $\boldsymbol{F}$. Therefore, for every $\Omega_{1} \in \mathcal{O}$, the Lagrangian $\mathscr{L}$ has a saddle point if and only if the saddle point equations

$$
\begin{array}{lll}
\text { Find } \boldsymbol{E} \in \boldsymbol{V}, & \partial_{\boldsymbol{F}} \mathscr{L}\left(\boldsymbol{E}, \boldsymbol{F}, \Omega_{1} ; \hat{\boldsymbol{F}}\right)=0 & \forall \hat{\boldsymbol{F}} \in \boldsymbol{V}, \\
\text { Find } \boldsymbol{F} \in \boldsymbol{V}, & \partial_{\boldsymbol{E}} \mathscr{L}\left(\boldsymbol{E}, \boldsymbol{F}, \Omega_{1} ; \hat{\boldsymbol{E}}\right)=0 & \forall \hat{\boldsymbol{E}} \in \boldsymbol{V},
\end{array}
$$

have a solution $(\boldsymbol{E}, \boldsymbol{F})$; see [11]. Note that equation (4.13) is the so-called state equation and coincides with (3.1) or equivalently with (4.11). According to Lemma 3.2, there exists a unique solution $\boldsymbol{E} \in \boldsymbol{V}$ of (3.1) and thus for (4.13). Similarly to Lemma 3.2, the Lax-Milgram lemma implies that the adjoint equation (4.14) admits a unique solution $\boldsymbol{F} \in \boldsymbol{V}$. Its explicit variational formulation is specified below. Thus, for every $\Omega_{1} \in \mathcal{O}$, the Lagrangian $\mathscr{L}$ admits a unique saddle point in $\boldsymbol{V} \times \boldsymbol{V}$. 
4.3. Differentiability of a saddle point. In order to study the shape differentiability of $\mathcal{J}\left(\Omega_{1}\right)$, we need to differentiate the saddle point (4.12). For the convenience of the reader, we repeat in this section an abstract result on differentiation of saddle point problems following [9]. Let us consider a functional

$$
f: Z \times Y \times[0, \tau] \rightarrow \mathbb{R}
$$

for some constant $\tau>0$ and Banach spaces $Z$ and $Y$. For each $t \in[0, \tau]$ define

$$
l(t):=\inf _{z \in Z} \sup _{y \in Y} f(z, y, t), \quad h(t):=\sup _{y \in Y} \inf _{z \in Z} f(z, y, t),
$$

and the associated sets

$$
\begin{aligned}
& Z(t):=\left\{z^{t} \in Z: \sup _{y \in Y} f\left(z^{t}, y, t\right)=l(t)\right\}, \\
& Y(t):=\left\{y^{t} \in Y: \inf _{z \in Z} f\left(z, y^{t}, t\right)=h(t)\right\} .
\end{aligned}
$$

Note that the inequality $h(t) \leq l(t)$ holds. If $l(t)=h(t)$ the set of saddle points is given by

$$
S(t):=Z(t) \times Y(t)
$$

We state a simplified version of a result of [8] derived from [6] which gives realistic conditions that allow to differentiate $l(t)$ at $t=0$. The main difficulty is to obtain conditions which allow to exchange the derivative with respect to $t$ and the inf-sup in (4.16).

TheOREM 4.3 (Correa and Seeger [6]). Let the Banach spaces $Z, Y$, the constant $\tau>0$, and the functional

$$
f: Z \times Y \times[0, \tau] \rightarrow \mathbb{R}
$$

be given. Assume that the following assumptions hold:

(H1) $S(t) \neq \emptyset$ for $0 \leq t \leq \tau$.

(H2) The partial derivative $\partial_{t} f(z, y, t)$ exists for all $(z, y, t) \in Z \times Y \times[0, \tau]$.

(H3) For any sequence $\left\{t_{n}\right\}_{n \in \mathbb{N}}$, with $t_{n} \rightarrow 0$, there exist a subsequence $\left\{t_{n_{k}}\right\}_{k \in \mathbb{N}}$ and $z^{0} \in Z(0)$, $z_{n_{k}} \in Z\left(t_{n_{k}}\right)$ such that for all $y \in Y(0)$,

$$
\lim _{t \searrow 0, k \rightarrow \infty} \partial_{t} f\left(z_{n_{k}}, y, t\right)=\partial_{t} f\left(z^{0}, y, 0\right) .
$$

(H4) For any sequence $\left\{t_{n}\right\}_{n \in \mathbb{N}}$, with $t_{n} \rightarrow 0$, there exist a subsequence $\left\{t_{n_{k}}\right\}_{k \in \mathbb{N}}$ and $y^{0} \in Y(0)$, $y_{n_{k}} \in Y\left(t_{n_{k}}\right)$ such that for all $z \in Z(0)$,

$$
\lim _{t \searrow 0, k \rightarrow \infty} \partial_{t} f\left(z, y_{n_{k}}, t\right)=\partial_{t} f\left(z, y^{0}, 0\right) .
$$

Then there exists $\left(z^{0}, y^{0}\right) \in Z(0) \times Y(0)$ such that

$$
\frac{d l}{d t}(0)=\partial_{t} f\left(z^{0}, y^{0}, 0\right)
$$


4.4. Function space parameterization. Let $\boldsymbol{\theta} \in \boldsymbol{\Theta}^{k}$, with $k \geq 2$, and $\Omega_{1} \in \mathcal{O}$ be given. We assume that $\boldsymbol{\theta}$ has a compact support in a $\mathcal{C}^{2,1}$-domain $\mathcal{D}$ satisfying $\bar{\Omega}_{1} \subset \mathcal{D}$ and $\overline{\mathcal{D}} \subset \Omega_{C}$, so that only $\Gamma_{1}:=\partial \Omega_{1}$ is affected by the transformation $T_{t}(\boldsymbol{\theta})$, i.e., we have

$$
T_{t}(\boldsymbol{\theta})\left(\Omega_{C}\right)=\Omega_{C} \quad \forall t \in[0, \tau] .
$$

We consider the family of domains $\Omega_{1}(t)=T_{t}(\boldsymbol{\theta})\left(\Omega_{1}\right)$ as defined in (4.2)-(4.3). In what follows, to simplify the notation, we write $T_{t}:=T_{t}(\boldsymbol{\theta})$.

In this section, we apply Theorem 4.3 to obtain the shape gradient of

$$
\mathcal{J}\left(\Omega_{1}(t)\right)=\left(\inf _{E \in \boldsymbol{V}} \sup _{F \in \boldsymbol{V}} \mathscr{L}\left(\boldsymbol{E}, \boldsymbol{F}, \Omega_{1}(t)\right)\right)+\kappa L\left(\sigma\left(\Omega_{1}(t)\right)\right) .
$$

As it is classical in shape optimization, the shape derivative $\left.\partial_{t} \mathcal{J}\left(\Omega_{1}(t)\right)\right|_{t=0}$ may be written as a boundary integral on $\Gamma_{1}$ including in particular the trace of $\boldsymbol{E} \cdot \overline{\boldsymbol{F}}$ on $\Gamma_{1}$, which requires higher regularity of $\boldsymbol{E}$ and $\boldsymbol{F}$ in $\Omega_{1}$ and $\mathcal{D} \backslash \overline{\Omega_{1}}$. This regularity is guaranteed by Theorem 3.4.

In order to differentiate $\mathscr{L}\left(\boldsymbol{E}, \boldsymbol{F}, \Omega_{1}(t)\right)$ with respect to $t$, the integrals in $\mathscr{L}\left(\boldsymbol{E}, \boldsymbol{F}, \Omega_{1}(t)\right)$ on the domains $\Omega_{0}(t)$ and $\Omega_{1}(t)$ need to be transported back to the fixed domains $\Omega_{0}$ and $\Omega_{1}$ using the transformation $T_{t}$. However, composing by $T_{t}$ inside the integrals creates terms $\boldsymbol{E} \circ T_{t}$ and $\boldsymbol{F} \circ T_{t}$ which might be nondifferentiable. To avoid this problem, we need to parameterize the space $\boldsymbol{V}$ by composing the elements of $\boldsymbol{V}$ with $T_{t}^{-1}$. However, unlike the case of $\boldsymbol{H}^{1}$, it is a well-known difficulty of shape optimization problems for Maxwell equations that this transformation modifies $\boldsymbol{V}$ globally [20,28], i.e.

$$
\boldsymbol{V} \neq\left\{\boldsymbol{\psi} \circ T_{t}^{-1}, \boldsymbol{\psi} \in \boldsymbol{V}\right\}
$$

Indeed, the transformation $T_{t}^{-1}$ which corresponds to a transport from $\Omega_{1}$ to $\Omega_{1}(t)$ modifies the curl operator as can be seen in the following formula (see [20, Section 3] for instance)

$$
\operatorname{curl} \psi=\xi(t) D T_{t}^{-1}\left(D T_{t}^{-*} \otimes D\left(\psi \circ T_{t}^{-1}\right) \circ T_{t}\right),
$$

where $D T_{t}: \mathbb{R}^{3} \rightarrow \mathbb{R}^{3 \times 3}$ is the Jacobian matrix function of $T_{t}, \xi(t)=\operatorname{det}\left(D T_{t}\right)$, and $\otimes$ the tensor product. Further, the notation $M^{*}$ denotes the transpose of a matrix $M$ and $M^{-*}=\left(M^{-1}\right)^{*}$. One observes on the right-hand side of (4.23) that $D\left(\boldsymbol{\psi} \circ T_{t}^{-1}\right)$ appears and $\boldsymbol{\psi} \circ T_{t}^{-1}$ must be in $\boldsymbol{H}^{1}$ for $\boldsymbol{\psi}$ to be in $\boldsymbol{V}$. This is a well-know issue when one tries to compute the shape derivative for Maxwell problems. To get around this problem, we consider the transformation

$$
\widetilde{\psi}=D T_{t}^{-*} \psi \circ T_{t}^{-1}
$$

According to [28, Corollary 3.58], we have the following identity

$$
(\widetilde{\operatorname{curl}} \widetilde{\psi}) \circ T_{t}=\xi(t)^{-1} D T_{t} \operatorname{curl} \psi,
$$

where $\widetilde{\text { curl }}$ denotes the operator with respect to the transformed variable $y=T_{t}(x)$. Therefore

$$
\operatorname{curl} \psi \in L^{2}(\Omega) \Longleftrightarrow \widetilde{\operatorname{curl}} \widetilde{\psi} \in L^{2}(\Omega) .
$$

Since $\boldsymbol{\theta} \equiv 0$ in $\Omega_{I}$, we clearly have $\widetilde{\boldsymbol{\psi}}=\boldsymbol{\psi}$ in $\Omega_{T} \cup \Omega_{R} \cup \Omega_{I}$, and finally we obtain

$$
\boldsymbol{V}=\left\{D T_{t}^{-*} \boldsymbol{\psi} \circ T_{t}^{-1}: \boldsymbol{\psi} \in \boldsymbol{V}\right\} .
$$


Using this reparameterization, we do not change the saddle point $\mathcal{J}\left(\Omega_{1}(t)\right)$ and so we may write

$$
\mathcal{J}\left(\Omega_{1}(t)\right)=\left(\inf _{\boldsymbol{E} \in \boldsymbol{V}} \sup _{\boldsymbol{F} \in \boldsymbol{V}} \widetilde{\mathscr{L}}(\boldsymbol{E}, \boldsymbol{F}, t)\right)+\kappa L\left(\sigma\left(\Omega_{1}(t)\right)\right)
$$

with

$$
\widetilde{\mathscr{L}}(\boldsymbol{E}, \boldsymbol{F}, t):=\mathscr{L}\left(D T_{t}^{-*} \boldsymbol{E} \circ T_{t}^{-1}, D T_{t}^{-*} \boldsymbol{F} \circ T_{t}^{-1}, \Omega_{1}(t)\right) .
$$

By the definition of $\mathscr{L}$, the explicit expression of $\widetilde{\mathscr{L}}(\boldsymbol{E}, \boldsymbol{F}, t)$ is obtained in the following form:

$$
\begin{aligned}
\widetilde{\mathscr{L}}(\boldsymbol{E}, \boldsymbol{F}, t) & =K(\boldsymbol{E})+\mathcal{R} e\left(\int_{\Omega} \mu^{-1} \widetilde{\boldsymbol{\operatorname { c u r l }}}\left(D T_{t}^{-*} \boldsymbol{E} \circ T_{t}^{-1}\right) \cdot \widetilde{\operatorname{curl}}\left(D T_{t}^{-*} \overline{\boldsymbol{F}} \circ T_{t}^{-1}\right)\right) \\
& +\mathcal{R} e\left(i \omega \sigma_{0} \int_{\Omega_{0}(t)}\left(D T_{t}^{-*} \boldsymbol{E} \circ T_{t}^{-1}\right) \cdot\left(D T_{t}^{-*} \overline{\boldsymbol{F}} \circ T_{t}^{-1}\right)\right) \\
& +\mathcal{R} e\left(i \omega \sigma_{1} \int_{\Omega_{1}(t)}\left(D T_{t}^{-*} \boldsymbol{E} \circ T_{t}^{-1}\right) \cdot\left(D T_{t}^{-*} \overline{\boldsymbol{F}} \circ T_{t}^{-1}\right)\right), \\
& +\mathcal{R} e\left(\int_{\Omega_{I}} \operatorname{div} \boldsymbol{E} \operatorname{div} \overline{\boldsymbol{F}}+i \omega \int_{\Omega_{T}} \boldsymbol{J} \cdot \overline{\boldsymbol{F}}\right) .
\end{aligned}
$$

where $\Omega_{0}(t):=\Omega_{C} \backslash \overline{\Omega_{1}(t)}$. Note that $K(\boldsymbol{E})$ and the integrals on $\Omega_{I}, \Omega_{T}$ and $\Omega_{R}$ in $\widetilde{\mathscr{L}}(\boldsymbol{E}, \boldsymbol{F}, t)$ are not affected by the transformation since $\boldsymbol{\theta} \equiv 0$ in $\Omega_{I}$. Using (4.24) and applying the change of variable $y=T_{t}(x)$ in (4.27)-(4.29) we get

$$
\begin{aligned}
\widetilde{\mathscr{L}}(\boldsymbol{E}, \boldsymbol{F}, t)=K(\boldsymbol{E}) & +\mathcal{R} e\left(\int_{\Omega} \mu^{-1} A(t) \operatorname{curl} \boldsymbol{E} \cdot \operatorname{curl} \overline{\boldsymbol{F}}+i \omega \sigma_{0} \int_{\Omega_{0}} C(t) \boldsymbol{E} \cdot \overline{\boldsymbol{F}}+i \omega \sigma_{1} \int_{\Omega_{1}} C(t) \boldsymbol{E} \cdot \overline{\boldsymbol{F}}\right) \\
& +\mathcal{R} e\left(i \omega \int_{\Omega_{T}} \boldsymbol{J} \cdot \overline{\boldsymbol{F}}+\int_{\Omega_{I}} \operatorname{div} \boldsymbol{E} \operatorname{div} \overline{\boldsymbol{F}}\right)
\end{aligned}
$$

where

$$
A(t):=|\xi(t)|^{-1} D T_{t}^{*} D T_{t}, \quad C(t):=|\xi(t)| D T_{t}^{-1} D T_{t}^{-*}=A(t)^{-1} .
$$

Notice that, for every $t \in[0, \tau]$, the matrix functions $A(t), C(t) \in \mathcal{C}^{k-1}\left(\mathbb{R}^{3}, \mathbb{R}^{3 \times 3}\right)$ are symmetric. Analogously to (4.13)-(4.14), the saddle point $\left(\boldsymbol{E}^{t}, \boldsymbol{F}^{t}\right)$ of $\widetilde{\mathscr{L}}(\boldsymbol{E}, \boldsymbol{F}, t)$ is defined as the solution of

Find $\boldsymbol{E}^{t} \in \boldsymbol{V}$ such that for all $\hat{\boldsymbol{F}} \in \boldsymbol{V}$ :

$$
\begin{aligned}
& \int_{\Omega} \mu^{-1} A(t) \operatorname{curl} \boldsymbol{E}^{t} \cdot \operatorname{curl} \overline{\hat{\boldsymbol{F}}}+i \omega \sigma_{0} \int_{\Omega_{0}} C(t) \boldsymbol{E}^{t} \cdot \overline{\hat{\boldsymbol{F}}} \\
& +i \omega \sigma_{1} \int_{\Omega_{1}} C(t) \boldsymbol{E}^{t} \cdot \overline{\hat{\boldsymbol{F}}}+\int_{\Omega_{I}} \operatorname{div} \boldsymbol{E}^{t} \operatorname{div} \overline{\hat{\boldsymbol{F}}}=-\int_{\Omega} i \omega \boldsymbol{J} \cdot \overline{\hat{\boldsymbol{F}}}
\end{aligned}
$$

Find $\boldsymbol{F}^{t} \in \boldsymbol{V}$ such that for all $\hat{\boldsymbol{E}} \in \boldsymbol{V}$ :

$$
\begin{aligned}
& \int_{\Omega} \mu^{-1} A(t) \operatorname{curl} \overline{\boldsymbol{F}^{t}} \cdot \operatorname{curl} \hat{\boldsymbol{E}}+i \omega \sigma_{0} \int_{\Omega_{0}} C(t) \overline{\boldsymbol{F}^{t}} \cdot \hat{\boldsymbol{E}} \\
& +i \omega \sigma_{1} \int_{\Omega_{1}} C(t) \overline{\boldsymbol{F}^{t}} \cdot \hat{\boldsymbol{E}}+\int_{\Omega_{I}} \operatorname{div} \overline{\boldsymbol{F}^{t}} \operatorname{div} \hat{\boldsymbol{E}}=-K^{\prime}\left(\boldsymbol{E}^{t}\right) \hat{\boldsymbol{E}}
\end{aligned}
$$


where

$$
\begin{aligned}
K^{\prime}\left(\boldsymbol{E}^{t}\right) \hat{\boldsymbol{E}} & =\sum_{k=1}^{n} \mathcal{R} e\left(\mathcal{M}^{k}\left(\boldsymbol{E}^{t}\right)-\mathcal{M}^{k, r e f}\right) \mathcal{R} e\left(\int_{R_{k}} \hat{\boldsymbol{E}} \cdot \mathbf{J}_{k}\right) \\
& +\sum_{k=1}^{n} \mathcal{I} m\left(\mathcal{M}^{k}\left(\boldsymbol{E}^{t}\right)-\mathcal{M}^{k, r e f}\right) \mathcal{I} m\left(\int_{R_{k}} \hat{\boldsymbol{E}} \cdot \mathbf{J}_{k}\right) .
\end{aligned}
$$

4.5. Domain expression of the shape gradient. In this section the shape derivative of $\mathcal{J}\left(\Omega_{1}(t)\right)$ expressed as a domain integral is obtained in Theorem 4.4 using the results of Section 4.3, which requires first to check the hypothesis of Theorem 4.3. In our case, the Banach spaces $Z, Y$ and the functional $f$ from Theorem 4.3 are chosen as

$$
Z=Y=\boldsymbol{V} \text { and } \quad f=\widetilde{\mathscr{L}}
$$

We start with (H1). Choose $\tau$ small enough such that $\xi(t)=|\xi(t)|$ for all $t \in[0, \tau]$. We have the following expansions which are valid in the norm of $\Theta^{k}$ :

$$
\begin{aligned}
\xi(t) & =1+t \operatorname{div}(\boldsymbol{\theta})+o(t), \\
D T_{t} & =I_{d}+t D \boldsymbol{\theta}+o(t), \\
D T_{t}^{-1} & =I_{d}-t D \boldsymbol{\theta}+o(t),
\end{aligned}
$$

where $I_{d} \in \mathbb{R}^{3 \times 3}$ denotes the identity matrix and $o\left(t_{n}\right) / t_{n} \rightarrow 0$ for $t_{n} \rightarrow 0, t_{n} \neq 0$ for all $n$. In view of (4.31) and (4.34)-(4.36) we get

$$
\begin{aligned}
& A(t)=I_{d}+t\left(-\operatorname{div}(\boldsymbol{\theta}) I_{d}+D \boldsymbol{\theta}+D \boldsymbol{\theta}^{*}\right)+o(t), \\
& C(t)=I_{d}+t\left(\operatorname{div}(\boldsymbol{\theta}) I_{d}-D \boldsymbol{\theta}-D \boldsymbol{\theta}^{*}\right)+o(t) .
\end{aligned}
$$

We observe that $A(t), C(t)$ are small perturbations of the identity in the $L^{\infty}$-norm. We choose now $\tau>0$ to be sufficiently small such that the matrix functions $A(t), C(t)$ are uniformly positive definite for every $t \in[0, \tau]$. Thus, analogously to Lemma 3.2, the Lax-Milgram lemma implies that the system (4.32)-(4.33) admits, for every $t \in[0, \tau]$, a unique solution $\left(\boldsymbol{E}^{t}, \boldsymbol{F}^{t}\right) \in \boldsymbol{V} \times \boldsymbol{V}$ satisfying

$$
\begin{aligned}
& \left\|\boldsymbol{E}^{t}\right\|_{\boldsymbol{V}} \leq c\|\boldsymbol{J}\|_{\boldsymbol{L}^{2}(\Omega)}, \\
& \left\|\boldsymbol{F}^{t}\right\|_{\boldsymbol{V}} \leq c\left(\left\|\boldsymbol{E}^{t}\right\|_{\boldsymbol{V}}+1\right),
\end{aligned}
$$

with a constant $c>0$ independent of $t$. Therefore, for every $t \in[0, \tau]$, we obtain $Z(t)=\left\{\boldsymbol{E}^{t}\right\} \neq \emptyset$, $Y(t)=\left\{\boldsymbol{F}^{t}\right\} \neq \emptyset$, and so

$$
S(t)=Z(t) \times Y(t) \neq \emptyset \quad \forall t \in[0, \tau]
$$

In conclusion, (H1) is satisfied.

To check (H2), we compute $\partial_{t} \widetilde{\mathscr{L}}(\boldsymbol{E}, \boldsymbol{F}, t)$. It is given by

$$
\partial_{t} \widetilde{\mathscr{L}}(\boldsymbol{E}, \boldsymbol{F}, t)=\mathcal{R} e\left(\int_{\Omega} \mu^{-1} A^{\prime}(t) \operatorname{curl} \boldsymbol{E} \cdot \operatorname{curl} \overline{\boldsymbol{F}}+i \omega \sigma_{0} \int_{\Omega_{0}} C^{\prime}(t) \boldsymbol{E} \cdot \overline{\boldsymbol{F}}+i \omega \sigma_{1} \int_{\Omega_{1}} C^{\prime}(t) \boldsymbol{E} \cdot \overline{\boldsymbol{F}}\right) .
$$


Define $\boldsymbol{\theta}_{t}(X):=\boldsymbol{\theta}\left(T_{t}(X)\right)$ and $B(t):=D T_{t}^{-*}$. The derivative of the inverse of a matrix $M$ in direction $Q$ is given by $D\left(M^{-1}\right)(Q)=-M^{-1} Q M$. Therefore we have

$$
B^{\prime}(t)=-B(t) D \boldsymbol{\theta}_{t}^{*} B(t), \quad \xi^{\prime}(t)=\operatorname{tr}\left(D \boldsymbol{\theta}_{t} B(t)^{*}\right) \xi(t) .
$$

Since $C(t)=\xi(t) B(t)^{*} B(t)$, using the chain rule yields

$$
\begin{aligned}
C^{\prime}(t) & =\xi^{\prime}(t) B(t)^{*} B(t)+\xi(t) B^{\prime}(t)^{*} B(t)+\xi(t) B(t)^{*} B^{\prime}(t) \\
& =\operatorname{tr}\left(D \boldsymbol{\theta}_{t} B(t)^{*}\right) C(t)-B(t)^{*} D \boldsymbol{\theta}_{t} C(t)-C(t) D \boldsymbol{\theta}_{t}^{*} B(t) .
\end{aligned}
$$

Since $A(t)=C(t)^{-1}$ we have

$$
\begin{aligned}
A^{\prime}(t) & =-A(t) C^{\prime}(t) A(t) \\
& =-A(t) \operatorname{tr}\left(D \boldsymbol{\theta}_{t} B(t)^{*}\right) C(t) A(t)+A(t) B(t)^{*} D \boldsymbol{\theta}_{t} C(t) A(t)+A(t) C(t) D \boldsymbol{\theta}_{t} B(t) A(t) \\
& =-A(t) \operatorname{tr}\left(D \boldsymbol{\theta}_{t} B(t)^{*}\right)+A(t) B(t)^{*} D \boldsymbol{\theta}_{t}+D \boldsymbol{\theta}_{t}^{*} B(t) A(t) .
\end{aligned}
$$

By the choice of $\boldsymbol{\theta} \in \boldsymbol{\Theta}^{k}$, with $k \geq 2$, the mappings $t \mapsto \boldsymbol{\theta}_{t}$ and $t \mapsto D \boldsymbol{\theta}_{t}$ are continuous on $[0, \tau]$ and consequently also $t \mapsto\left(B(t), A(t), A^{\prime}(t), C^{\prime}(t)\right)$. For this reason, $\partial_{t} \widetilde{\mathscr{L}}(\boldsymbol{E}, \boldsymbol{F}, t)$ exists everywhere in $[0, \tau]$ for all $\boldsymbol{E}, \boldsymbol{F} \in \boldsymbol{V}$. Therefore, the condition (H2) is satisfied.

Now, we check (H3) and (H4). According to (4.37)-(4.38), the sequence $\left\{\left(\boldsymbol{E}^{t}, \boldsymbol{F}^{t}\right)\right\}_{0 \leq t \leq \tau}$ is bounded in $\boldsymbol{V} \times \boldsymbol{V}$. Consequently, by possibly extracting a subsequence, we find a pair $(\widetilde{\boldsymbol{E}}, \widetilde{\boldsymbol{F}})$ in $\boldsymbol{V} \times \boldsymbol{V}$ such that

$$
\boldsymbol{E}^{t} \rightarrow \widetilde{\boldsymbol{E}} \quad \boldsymbol{F}^{t} \rightarrow \widetilde{\boldsymbol{F}} \quad \text { weakly in } \boldsymbol{V} \text { as } t \rightarrow 0 .
$$

In addition, we have the following uniform convergences

$$
A(t) \rightarrow I_{d} \quad C(t) \rightarrow I_{d} \quad \text { strongly in } \mathcal{C}^{k-1}\left(\mathbb{R}^{3}, \mathbb{R}^{3 \times 3}\right) \text { as } t \rightarrow 0 .
$$

Therefore, passing to the limit $t \rightarrow 0$ in the variational equations (4.32)-(4.33), it follows from (4.40)-(4.41) that the pair $(\widetilde{\boldsymbol{E}}, \boldsymbol{F})$ is exactly the solution of

Find $\boldsymbol{E} \in \boldsymbol{V}$ such that for all $\hat{\boldsymbol{F}} \in \boldsymbol{V}$ :

$$
\begin{aligned}
& \int_{\Omega} \mu^{-1} \operatorname{curl} \boldsymbol{E} \cdot \operatorname{curl} \overline{\hat{\boldsymbol{F}}}+i \omega \sigma_{0} \int_{\Omega_{0}} \boldsymbol{E} \cdot \overline{\hat{\boldsymbol{F}}} \\
& +i \omega \sigma_{1} \int_{\Omega_{1}} \boldsymbol{E} \cdot \overline{\hat{\boldsymbol{F}}}+\int_{\Omega_{I}} \operatorname{div} \boldsymbol{E} \operatorname{div} \overline{\hat{\boldsymbol{F}}}=-\int_{\Omega} i \omega \boldsymbol{J} \cdot \overline{\hat{\boldsymbol{F}}}
\end{aligned}
$$

and

$$
\begin{aligned}
& \text { Find } \boldsymbol{F} \in \boldsymbol{V} \text { such that for all } \hat{\boldsymbol{E}} \in \boldsymbol{V} \text { : } \\
& \int_{\Omega} \mu^{-1} \operatorname{curl} \overline{\boldsymbol{F}} \cdot \operatorname{curl} \hat{\boldsymbol{E}}+i \omega \sigma_{0} \int_{\Omega_{0}} \overline{\boldsymbol{F}} \cdot \hat{\boldsymbol{E}} \\
& +i \omega \sigma_{1} \int_{\Omega_{1}} \overline{\boldsymbol{F}} \cdot \hat{\boldsymbol{E}}+\int_{\Omega_{I}} \operatorname{div} \overline{\boldsymbol{F}} \operatorname{div} \hat{\boldsymbol{E}}=-K^{\prime}(\boldsymbol{E}) \hat{\boldsymbol{E}} .
\end{aligned}
$$

In other words, the pair $(\widetilde{\boldsymbol{E}}, \widetilde{\boldsymbol{F}}) \in \boldsymbol{V} \times \boldsymbol{V}$ is the solution of the saddle point equations (4.13)-(4.14), or equivalently it is the unique saddle point of $\mathscr{L}$. Therefore, since $\widetilde{\mathscr{L}}(\cdot, \cdot, 0)=\mathscr{L}\left(\cdot, \cdot, \Omega_{1}\right)$, we obtain

$$
\widetilde{\boldsymbol{E}} \in Z(0) \text { and } \widetilde{\boldsymbol{F}} \in Y(0) .
$$


Now for every fixed $\boldsymbol{F} \in \boldsymbol{V}$, the mapping $(t, \boldsymbol{E}) \rightarrow \partial_{t} \widetilde{\mathscr{L}}(\boldsymbol{E}, \boldsymbol{F}, t)$ is strongly continuous in $t$ and weakly continuous in $\boldsymbol{E}$. Similarly, for every fixed $\boldsymbol{E} \in \boldsymbol{V}$, the mapping $(t, \boldsymbol{F}) \rightarrow \partial_{t} \widetilde{\mathscr{L}}(\boldsymbol{E}, \boldsymbol{F}, t)$ is strongly continuous in $t$ and weakly continuous in $\boldsymbol{F}$ in view of the above expression of $\partial_{t} \widetilde{\mathscr{L}}(\boldsymbol{E}, \boldsymbol{F}, t)$. Therefore we have the convergences:

$$
\begin{aligned}
\lim _{t \searrow 0, k \rightarrow \infty} \partial_{t} \widetilde{\mathscr{L}}\left(\boldsymbol{E}^{t_{n_{k}}}, \boldsymbol{F}, t\right) & =\partial_{t} \widetilde{\mathscr{L}}(\boldsymbol{E}, \boldsymbol{F}, 0), \\
\lim _{t \searrow 0, k \rightarrow \infty} \partial_{t} \widetilde{\mathscr{L}}\left(\boldsymbol{E}, \boldsymbol{F}^{t_{n_{k}}}, t\right) & =\partial_{t} \widetilde{\mathscr{L}}(\boldsymbol{E}, \boldsymbol{F}, 0) .
\end{aligned}
$$

Thus, assumptions (H3) and (H4) are satisfied. From this we conclude that all assumptions of Theorem 4.3 are satisfied.

It is a standard result $[9,19,33]$ in shape optimization that

$$
\partial_{t} L\left(\sigma\left(\Omega_{1}(t)\right)\right)_{\mid t=0}=\left|\sigma_{0}-\sigma_{1}\right| \int_{\Gamma_{1}} \mathcal{H}_{1} \boldsymbol{\theta} \cdot \boldsymbol{n}_{1} d \Gamma
$$

where $\mathcal{H}_{1}$ is the curvature associated with the boundary of the domain $\Omega_{1}$. The curvature $\mathcal{H}_{1}$ has regularity $\mathcal{C}^{0,1}$ for $\Omega_{1} \in \mathcal{O}$.

Thus, we arrive at the following formula for the shape derivative:

THEOREM 4.4. Let $\boldsymbol{\theta} \in \boldsymbol{\Theta}^{k}$, with $k \geq 2$, and $\Omega_{1} \in \mathcal{O}$ be given. Assume that $\boldsymbol{\theta}$ has compact support in $a \mathcal{C}^{2,1}$-domain $\mathcal{D}$ satisfying $\bar{\Omega}_{1} \subset \mathcal{D}$ and $\overline{\mathcal{D}} \subset \Omega_{C}$. Then,

$$
\begin{aligned}
d \mathcal{J}\left(\Omega_{1} ; \boldsymbol{\theta}\right) & =\partial_{t} \widetilde{\mathscr{L}}(\boldsymbol{E}, \boldsymbol{F}, 0)+\kappa \partial_{t} L\left(\sigma\left(\Omega_{1}(t)\right)\right) \\
=\mathcal{R} e & \left(\int_{\Omega} \mu^{-1} A^{\prime}(0) \operatorname{curl} \boldsymbol{E} \cdot \operatorname{curl} \overline{\boldsymbol{F}}+i \omega \sigma_{0} \int_{\Omega_{0}} C^{\prime}(0) \boldsymbol{E} \cdot \overline{\boldsymbol{F}}+i \omega \sigma_{1} \int_{\Omega_{1}} C^{\prime}(0) \boldsymbol{E} \cdot \overline{\boldsymbol{F}}\right) \\
& +\kappa\left|\sigma_{0}-\sigma_{1}\right| \int_{\Gamma_{1}} \mathcal{H}_{1} \boldsymbol{\theta} \cdot \boldsymbol{n}_{1} d \Gamma .
\end{aligned}
$$

with

$$
C^{\prime}(0)=-A^{\prime}(0)=\operatorname{div}\left(\boldsymbol{\theta}_{0}\right)-D \boldsymbol{\theta}_{0}-D \boldsymbol{\theta}_{0}^{*}
$$

and where the pair $(\boldsymbol{E}, \boldsymbol{F}) \in \boldsymbol{V} \times \boldsymbol{V}$ is the solution of (4.42)-(4.43).

4.6. Boundary expression of the shape gradient. Expression (4.45) of the shape gradient is constituted of domain integrals which are well-defined for $(\boldsymbol{E}, \boldsymbol{F}) \in \boldsymbol{V} \times \boldsymbol{V}$. It is readily seen that the mapping

$$
\boldsymbol{\Theta}^{k} \ni \boldsymbol{\theta} \mapsto d \mathcal{J}\left(\Omega_{1} ; \boldsymbol{\theta}\right) \in \mathbb{R}
$$

is linear and continuous. So by the structure theorem [9, Chapter 8, Theorem 3.5], if $\Gamma_{1}$ is of class $\mathcal{C}^{k+1}$, then $\boldsymbol{n}_{1}$ is in $\mathcal{C}^{k}\left(\Gamma_{1} ; \mathbb{R}^{3}\right)$ and there exists a scalar distribution $g\left(\Gamma_{1}\right) \in \mathcal{C}^{k}\left(\Gamma_{1}\right)^{\prime}$ such that

$$
d \mathcal{J}\left(\Omega_{1} ; \boldsymbol{\theta}\right)=\left\langle g\left(\Gamma_{1}\right), \boldsymbol{\theta} \cdot \boldsymbol{n}_{1}\right\rangle_{\mathcal{C}^{k}\left(\Gamma_{1}\right)} .
$$

In the case of $g\left(\Gamma_{1}\right) \in L^{1}\left(\Gamma_{1}\right)$, we also have the following integral representation:

$$
d \mathcal{J}\left(\Omega_{1} ; \boldsymbol{\theta}\right)=\int_{\Gamma_{1}} g\left(\Gamma_{1}\right) \boldsymbol{\theta} \cdot \boldsymbol{n}_{1} d \Gamma_{1} .
$$


Our goal now is to verify that $g\left(\Gamma_{1}\right) \in L^{1}\left(\Gamma_{1}\right)$ and to provide an explicit expression for the function $g\left(\Gamma_{1}\right)$. For this purpose we make use of the following result [19, Corollary 5.2.3]:

THEOREM 4.5 (Hadamard formula). Let $\boldsymbol{\theta} \in \boldsymbol{\Theta}^{k}, k \geq 1$, and $\left[0, \tau\left[\ni t \rightarrow \phi(t, \cdot) \in L^{1}\left(\mathcal{D}_{t}\right)\right.\right.$ where $\mathcal{D}_{t}:=T_{t}(\mathcal{D})$ and $\mathcal{D}$ is a given measurable bounded set. Assume

$$
\left[0, \tau\left[\ni t \rightarrow \Phi(t, \cdot):=\phi\left(t, T_{t}(\cdot)\right) \in L^{1}(\mathcal{D}) \text { is differentiable at } 0 .\right.\right.
$$

and that there exists a linear and continuous extension operator $P: L^{1}(\Omega) \rightarrow L^{1}\left(\mathbb{R}^{3}\right)$ such that

$$
P(\phi(0, \cdot)) \in W^{1,1}\left(\mathbb{R}^{3}\right) .
$$

Then there exists an extension $\left[0, \tau\left[\ni t \rightarrow \widetilde{\phi}(t, \cdot) \in L^{1}\left(\mathbb{R}^{3}\right)\right.\right.$ of $t \rightarrow \phi(t, \cdot)$ which is differentiable at zero with

$$
\widetilde{\phi}^{\prime}(0, \cdot)=\Phi^{\prime}(0, \cdot)-\nabla P(\phi(0, \cdot)) \cdot \boldsymbol{\theta}
$$

In addition

$$
t \rightarrow I(t):=\int_{\mathcal{D}_{t}} \phi(t, x) d x
$$

is differentiable at 0 , and setting $\phi^{\prime}(0, x):=\widetilde{\phi}^{\prime}(0, x)$ we get

$$
I^{\prime}(0)=\int_{\mathcal{D}} \operatorname{div}(\phi(0, x) \boldsymbol{\theta})+\phi^{\prime}(0, x) d x .
$$

If $\mathcal{D}$ is Lipschitz, then

$$
I^{\prime}(0)=\int_{\partial \mathcal{D}} \phi(0, x) \boldsymbol{\theta} \cdot \boldsymbol{n} d \Gamma+\int_{\mathcal{D}} \phi^{\prime}(0, x) d x
$$

where $\boldsymbol{n}$ is the outward unit normal vector to $\mathcal{D}$.

In order to obtain the boundary expression of the shape gradient, the idea is to differentiate the expression (4.27)-(4.30) directly using Theorem 4.5 and then apply Theorem 4.3. We consider the integrals in (4.27)-(4.30) separately. The terms $K(\boldsymbol{E})$ in (4.27) and the term (4.30) are clearly independent of $t$. For the other terms, we make use of Theorem 4.5 with $\mathcal{D}_{t}=\Omega_{0}(t)$ and $\mathcal{D}_{t}=\Omega_{1}(t)$ successively. First we choose

$$
\phi(t, x)=\left(D T_{t}^{-*} \boldsymbol{E} \circ T_{t}^{-1}(x)\right) \cdot\left(D T_{t}^{-*} \overline{\boldsymbol{F}} \circ T_{t}^{-1}(x)\right) \in L^{1}\left(\Omega_{1}(t)\right) .
$$

Let us now verify the hypothesis of Theorem 4.5. First of all, for $\boldsymbol{\theta} \in \boldsymbol{\Theta}^{k}$, with $k \geq 1$, the mapping $t \mapsto T_{t}$ is of class $\mathcal{C}^{1}\left([0, \tau], \mathbf{\Theta}^{k}\right)$ (see [9, Chapter 7, Theorem 4.3]). Also, the mapping $t \mapsto D T_{t}$ is welldefined as an element in $\mathcal{C}^{1}\left([0, \tau], \mathcal{C}^{k-1}\left(\mathbb{R}^{3}, \mathbb{R}^{3 \times 3}\right)\right)$. In view of (4.39), for $t$ small enough, we also have that the mapping $t \mapsto D T_{t}^{-1}$ is of class $\mathcal{C}^{1}\left([0, \tau], \mathcal{C}^{k-1}\left(\mathbb{R}^{3}, \mathbb{R}^{3 \times 3}\right)\right)$.

Since $(\boldsymbol{E}, \boldsymbol{F}) \in \boldsymbol{V} \times \boldsymbol{V}$, we obtain

$$
\phi\left(t, T_{t}(\cdot)\right)=\Phi(t, \cdot)=\left(D T_{t}^{-*} \boldsymbol{E}\right) \cdot\left(D T_{t}^{-*} \overline{\boldsymbol{F}}\right) \in L^{1}\left(\Omega_{1}\right)
$$

and $\Phi(t, \cdot)$ is differentiable at $t=0$ with

$$
\Phi^{\prime}(0, \cdot)=-\left(D \boldsymbol{\theta}^{*} \boldsymbol{E}\right) \cdot \overline{\boldsymbol{F}}-\boldsymbol{E} \cdot\left(D \boldsymbol{\theta}^{*} \overline{\boldsymbol{F}}\right) \in L^{1}\left(\Omega_{1}\right) .
$$


We also have $\phi(0, \cdot)=\boldsymbol{E} \cdot \overline{\boldsymbol{F}}$. According to Theorem 3.4, we have $\boldsymbol{E}_{\mid \Omega_{1}} \in \boldsymbol{H}^{2}\left(\Omega_{1}\right)$. Similarly to Theorem 3.4, we can show that $\boldsymbol{F}_{\mid \Omega_{1}} \in \boldsymbol{H}^{2}\left(\Omega_{1}\right)$. Therefore, $(\boldsymbol{E} \cdot \overline{\boldsymbol{F}})_{\mid \Omega_{1}} \in W^{1,1}\left(\Omega_{1}\right)$ holds by the Sobolev embedding theorem. A linear and continuous extension $P: L^{1}\left(\Omega_{1}\right) \rightarrow L^{1}\left(\mathbb{R}^{3}\right)$ such that $P\left((\boldsymbol{E} \cdot \overline{\boldsymbol{F}})_{\mid \Omega_{1}}\right) \in W^{1,1}\left(\mathbb{R}^{3}\right)$ exists as long as $\Omega_{1}$ is of class $\mathcal{C}^{1}$; see [4]. Thus, since $\Omega_{1} \in \mathcal{O}$, we may apply Theorem 4.5 with

$$
I_{1}(t):=\int_{\Omega_{1}(t)} \phi(t, x) d x
$$

Also, we may use (4.51) to obtain

$$
I_{1}^{\prime}(0)=\int_{\Gamma_{1}} \boldsymbol{E} \cdot \overline{\boldsymbol{F}} \boldsymbol{\theta} \cdot \boldsymbol{n}_{1} d \Gamma+\int_{\Omega_{1}} \dot{\boldsymbol{E}} \cdot \overline{\boldsymbol{F}}+\boldsymbol{E} \cdot \dot{\overline{\boldsymbol{F}}} d x
$$

where

$$
\begin{gathered}
\dot{\boldsymbol{E}}:=\left.\frac{d}{d t} D T_{t}^{-*} \boldsymbol{E} \circ T_{t}^{-1}\right|_{t=0}=-D \boldsymbol{E} \boldsymbol{\theta}-D \boldsymbol{\theta}^{*} \boldsymbol{E}, \\
\dot{\overline{\boldsymbol{F}}}:=\left.\frac{d}{d t} D T_{t}^{-*} \overline{\boldsymbol{F}} \circ T_{t}^{-1}\right|_{t=0}=-D \overline{\boldsymbol{F}} \boldsymbol{\theta}-D \boldsymbol{\theta}^{*} \overline{\boldsymbol{F}}
\end{gathered}
$$

Note that we have used the fact that $\left.\nabla(\boldsymbol{E} \cdot \overline{\boldsymbol{F}})\right|_{\Omega_{1}}=\nabla P\left((\boldsymbol{E} \cdot \overline{\boldsymbol{F}})_{\mid \Omega_{1}}\right)$.

In a similar way, we apply Theorem 4.5 with

$$
\begin{aligned}
I_{0}(t) & =\int_{\Omega_{0}(t)} \phi(t, x) d x \quad \text { and } \\
\phi(t, x) & =\left(D T_{t}^{-*} \boldsymbol{E} \circ T_{t}^{-1}(x)\right) \cdot\left(D T_{t}^{-*} \overline{\boldsymbol{F}} \circ T_{t}^{-1}(x)\right) \in L^{1}\left(\Omega_{0}(t)\right) .
\end{aligned}
$$

Then we obtain

$$
I_{0}^{\prime}(0)=\int_{\Gamma_{1}} \boldsymbol{E} \cdot \overline{\boldsymbol{F}} \boldsymbol{\theta} \cdot \boldsymbol{n}_{0} d \Gamma+\int_{\Omega_{0}} \dot{\boldsymbol{E}} \cdot \overline{\boldsymbol{F}}+\boldsymbol{E} \cdot \dot{\overline{\boldsymbol{F}}} d x
$$

where $\boldsymbol{n}_{0}$ is the outward unit normal vector to $\Omega_{0}$.

To compute the derivative of the integral in (4.27), we split it into three integrals on $\Omega_{I} \cup \Omega_{T}, \Omega_{1}(t)$ and $\Omega_{0}(t)$. The derivative of the integral on $\Omega_{I} \cup \Omega_{T}$ is zero since $\boldsymbol{\theta}$ has compact support in $\Omega_{C}$. For the integrals on $\Omega_{0}(t)$ and $\Omega_{1}(t)$, we apply Theorem 4.5. We take

$$
\phi(t, x)=\widetilde{\boldsymbol{\operatorname { c u r l }}}\left(D T_{t}^{-*} \boldsymbol{E} \circ T_{t}^{-1}\right)(x) \cdot \widetilde{\mathbf{c u r l}}\left(D T_{t}^{-*} \overline{\boldsymbol{F}} \circ T_{t}^{-1}\right)(x) .
$$

In view of (4.25), we have

$$
\phi\left(t, T_{t}(\cdot)\right)=: \Phi(t, \cdot)=\widetilde{\mathbf{\operatorname { c u r l }}}\left(D T_{t}^{-*} \boldsymbol{E} \circ T_{t}^{-1}\right)\left(T_{t}(\cdot)\right) \cdot \widetilde{\boldsymbol{\operatorname { c u r l }}}\left(D T_{t}^{-*} \overline{\boldsymbol{F}} \circ T_{t}^{-1}\right)\left(T_{t}(\cdot)\right) \in L^{1}\left(\Omega_{1}\right) .
$$

According to Theorem 3.4, $\boldsymbol{E}, \boldsymbol{F}$ enjoy higher regularity in $\boldsymbol{H}^{2}\left(\Omega_{1}\right)$. Therefore, $\dot{\boldsymbol{E}}, \dot{\boldsymbol{F}}$ are in $\boldsymbol{H}^{1}\left(\Omega_{1}\right)$ and the time-derivative of $\Phi(t, x)$ at $t=0$ is given by

$$
\Phi^{\prime}(0, \cdot)=\operatorname{curl} \dot{\boldsymbol{E}} \cdot \operatorname{curl} \overline{\boldsymbol{F}}+\operatorname{curl} \boldsymbol{E} \cdot \operatorname{curl} \dot{\overline{\boldsymbol{F}}}+D[\operatorname{curl}(\boldsymbol{E})] \boldsymbol{\theta} \cdot \operatorname{curl}(\overline{\boldsymbol{F}})+\operatorname{curl}(\boldsymbol{E}) \cdot D[\operatorname{curl}(\overline{\boldsymbol{F}})] \boldsymbol{\theta} .
$$


Thus with $\boldsymbol{\theta} \in \mathbf{\Theta}^{k}$ we get $\Phi^{\prime}(0, \cdot) \in L^{1}\left(\Omega_{1}\right)$. We also have

$$
\begin{aligned}
\nabla P\left((\operatorname{curl} \boldsymbol{E} \cdot \operatorname{curl} \overline{\boldsymbol{F}})_{\mid \Omega_{1}}\right) & =\nabla(\operatorname{curl} \boldsymbol{E} \cdot \operatorname{curl} \overline{\boldsymbol{F}})_{\mid \Omega_{1}} \\
& =-D[\operatorname{curl}(\boldsymbol{E})] \boldsymbol{\theta} \cdot \operatorname{curl}(\overline{\boldsymbol{F}})-\operatorname{curl}(\boldsymbol{E}) \cdot D[\operatorname{curl}(\overline{\boldsymbol{F}})] \boldsymbol{\theta}
\end{aligned}
$$

which yields

$$
\phi^{\prime}(0, \cdot)=\operatorname{curl} \dot{\boldsymbol{E}} \cdot \operatorname{curl} \overline{\boldsymbol{F}}+\operatorname{curl} \boldsymbol{E} \cdot \operatorname{curl} \dot{\overline{\boldsymbol{F}}}
$$

Defining

$$
I_{\text {curl }, i}(t):=\int_{\Omega_{i}(t)} \phi(t, x) d x, \quad i=0,1
$$

we get

$$
I_{\operatorname{curl}, i}^{\prime}(0)=\int_{\partial \Omega_{i}}(\operatorname{curl} \boldsymbol{E} \cdot \operatorname{curl} \overline{\boldsymbol{F}}) \boldsymbol{\theta} \cdot \boldsymbol{n}_{i} d \Gamma+\int_{\Omega_{i}} \operatorname{curl} \dot{\boldsymbol{E}} \cdot \operatorname{curl} \overline{\boldsymbol{F}}+\operatorname{curl} \boldsymbol{E} \cdot \operatorname{curl} \dot{\overline{\boldsymbol{F}}} d x, \quad i=0,1 .
$$

According to Lemma 3.3, $\operatorname{curl} \boldsymbol{E}$ and $\operatorname{curl} \overline{\boldsymbol{F}}$ are in $\boldsymbol{H}^{1}\left(\Omega_{C}\right)$. Thus, the above integral on $\partial \Omega_{i}$ is welldefined and

$$
\int_{\partial \Omega_{0}}(\operatorname{curl} \boldsymbol{E} \cdot \operatorname{curl} \overline{\boldsymbol{F}}) \boldsymbol{\theta} \cdot \boldsymbol{n}_{0} d \Gamma=-\int_{\partial \Omega_{1}}(\operatorname{curl} \boldsymbol{E} \cdot \operatorname{curl} \overline{\boldsymbol{F}}) \boldsymbol{\theta} \cdot \boldsymbol{n}_{1} d \Gamma .
$$

Now we check that $\dot{\boldsymbol{E}}$ and $\dot{\boldsymbol{F}}$ are in $\boldsymbol{V}$. Indeed we have

$$
\begin{aligned}
\operatorname{curl} \dot{\boldsymbol{E}} & =\operatorname{curl}\left(-D \boldsymbol{E} \boldsymbol{\theta}-D \boldsymbol{\theta}^{*} \boldsymbol{E}\right) \\
& =-(D \operatorname{curl} \boldsymbol{E}) \boldsymbol{\theta}+(D \boldsymbol{\theta}-(\operatorname{div} \boldsymbol{\theta}) I) \operatorname{curl} \boldsymbol{E}
\end{aligned}
$$

Since $\boldsymbol{E} \in \boldsymbol{V}$ and by Lemma 3.3, we get curl $\dot{\boldsymbol{E}} \in \boldsymbol{L}^{2}(\Omega)$ and consequently $\dot{\boldsymbol{E}} \in \boldsymbol{V}$. Since $\dot{\boldsymbol{E}}$ and $\dot{\overline{\boldsymbol{F}}}$ have compact support in $\Omega_{C}$ and taking (4.53) into account, we obtain

$$
\begin{aligned}
I_{\text {curl }, 1}^{\prime}(0)+I_{\operatorname{curl}, 2}^{\prime}(0) & =\int_{\Omega_{C}} \operatorname{curl} \dot{\boldsymbol{E}} \cdot \operatorname{curl} \overline{\boldsymbol{F}}+\operatorname{curl} \boldsymbol{E} \cdot \operatorname{curl} \dot{\overline{\boldsymbol{F}}} d x \\
& =\int_{\Omega} \operatorname{curl} \dot{\boldsymbol{E}} \cdot \operatorname{curl} \overline{\boldsymbol{F}}+\operatorname{curl} \boldsymbol{E} \cdot \operatorname{curl} \dot{\boldsymbol{F}} d x
\end{aligned}
$$

Gathering the previous results we get

$$
\begin{aligned}
\partial_{t} \widetilde{\mathscr{L}}(\boldsymbol{E}, \boldsymbol{F}, 0) & =\mathcal{R} e\left(\int_{\Omega} \mu^{-1} \operatorname{curl} \dot{\boldsymbol{E}} \cdot \operatorname{curl} \overline{\boldsymbol{F}} d x+i \omega \int_{\Omega_{0}} \sigma_{0} \dot{\boldsymbol{E}} \cdot \overline{\boldsymbol{F}} d x+i \omega \int_{\Omega_{1}} \sigma_{1} \dot{\boldsymbol{E}} \cdot \overline{\boldsymbol{F}} d x\right) \\
& +\mathcal{R} e\left(\int_{\Omega} \mu^{-1} \operatorname{curl} \boldsymbol{E} \cdot \operatorname{curl} \dot{\boldsymbol{F}} d x+i \omega \int_{\Omega_{0}} \sigma_{0} \boldsymbol{E} \cdot \dot{\boldsymbol{F}} d x+i \omega \int_{\Omega_{1}} \sigma_{1} \boldsymbol{E} \cdot \dot{\boldsymbol{F}} d x\right) \\
& +\mathcal{R} e\left(\int_{\Gamma_{1}} i \omega \llbracket \sigma \boldsymbol{E} \cdot \overline{\boldsymbol{F}} \rrbracket_{\Gamma_{1}} \boldsymbol{\theta} \cdot \boldsymbol{n}_{1} d \Gamma\right)
\end{aligned}
$$


where

$$
\llbracket \psi \rrbracket_{\Gamma_{1}}(x)=\lim _{y \in \Omega_{1}, y \rightarrow x} \psi(y)-\lim _{y \in \Omega \backslash \overline{\Omega_{1}}, y \rightarrow x} \psi(y)
$$

is the jump over $\Gamma_{1}$ of a function $\psi$ at $x \in \Gamma_{1}$. The expressions on the right hand sides of the equalities (4.54) and (4.55) vanish since they correspond to the variational formulations for the state and the adjoint (4.42) and (4.43) with the test functions $\dot{\boldsymbol{E}}$ and $\dot{\boldsymbol{F}} \in \boldsymbol{V}$ where we also use the fact that $\dot{\boldsymbol{E}}$ and $\dot{\boldsymbol{F}}$ have compact support in $\Omega_{C}$.

Finally Theorem 4.3 gives the shape derivative

$$
d \mathcal{J}\left(\Omega_{1} ; \boldsymbol{\theta}\right)=\partial_{t} \widetilde{\mathscr{L}}(\boldsymbol{E}, \boldsymbol{F}, 0)+\kappa \partial_{t} L\left(\sigma\left(\Omega_{1}(t)\right)\right)_{\mid t=0}
$$

Summarizing the previous results, we obtain the following theorem:

THEOREM 4.6. Let $\boldsymbol{\theta} \in \boldsymbol{\Theta}^{k}$, with $k \geq 1$, and $\Omega_{1} \in \mathcal{O}$ be given. Then the shape derivative of $\mathcal{J}$ at $\Omega_{1}$ in the direction $\boldsymbol{\theta}$ is given by

$$
d \mathcal{J}\left(\Omega_{1} ; \boldsymbol{\theta}\right)=\int_{\Gamma_{1}}\left[\mathcal{R} e\left(i \omega \llbracket \sigma \boldsymbol{E} \cdot \overline{\boldsymbol{F}} \rrbracket_{\Gamma_{1}}\right)+\kappa\left|\sigma_{0}-\sigma_{1}\right| \mathcal{H}_{1}\right] \boldsymbol{\theta} \cdot \boldsymbol{n}_{1} d \Gamma
$$

where $(\boldsymbol{E}, \boldsymbol{F}) \in \boldsymbol{V} \times \boldsymbol{V}$ is the solution of (4.42)-(4.43) and $\mathcal{H}_{1}$ is the curvature of $\Omega_{1}$.

5. Conclusions. Magnetic induction tomography (MIT) is a promising non-invasive imaging modality. The time harmonic eddy-current system formulated in terms of the electric field represents a suitable approximation of the full Maxwell system in a low frequency regime. It serves as a proper state system in an output least squares formulation for identifying hidden inclusions from measurements of the electric field in receiver coils surrounding the region of interest. The sharp interface formulation proposed in this paper considers, in addition to conductivity values, the shape of the hidden inclusions as the unknown. This puts the problem into the realm of shape optimization and requires shape sensitivity analysis for obtaining a shape gradient related descent direction for the numerical solution of the problem or to decide whether a given set of inclusions is first-order optimal.

To establish the domain and boundary form of the shape gradient, we have adapted a saddle point approach introduced in [8]. An interesting feature of our result, which is specific to the Maxwell equations, is the use of an unusual function space parameterization when differentiating the inf-sup of the Lagrangian. While the domain form of the shape gradient can be used as the basis for, e.g., a level-set based shape optimization algorithm for iteratively solving the output least squares problem, the well-known HadamardZolesio structure theorem suggests to rather use a boundary form of the shape gradient. However, for obtaining the latter in the present context higher regularity of the electric field had to be established. The corresponding boundary expression has the advantage of yielding lower dimensional quantities in numerical realizations. Structurally, it is composed of the real part of the product of the electric field and the associated adjoint state plus a weighted curvature term, where the weight accounts for conductivity differences in different regions within the domain of interest. While the latter term has a curve shortening (in 2D) or surface diminishing (in 3D) effect and results from regularization, the former is due to the quadratic misfit functional in the overall objective. Both, the domain as well as the boundary expression are suitable for being implemented in a shape sensitivity based minimization scheme.

As our current investigation has an intrinsic 3D character in connection with MIT, the efficient numerical treatment of the problem is an interesting, yet challenging task in its own right. Based on the theoretical findings of this paper, the design and implementation of a level-set based shape optimization method a future research task. 
Another aspect, which is open for future research is the computation of topological sensitivities for the underlying identification problems. The associated topological derivative is useful for detecting the topological distribution of hidden objects and may be used for initializing the shape sensitivity based method addressed above.

\section{REFERENCES}

[1] R. A. Adams and J. J. F. Fournier. Sobolev spaces, volume 140 of Pure and Applied Mathematics (Amsterdam). Elsevier/Academic Press, Amsterdam, second edition, 2003.

[2] A. Alonso Rodríguez and A. Valli. Eddy current approximation of Maxwell equations, volume 4 of MS\& A. Modeling, Simulation and Applications. Springer-Verlag Italia, Milan, 2010. Theory, algorithms and applications.

[3] Z. Belhachmi and H. Meftahi. Shape sensitivity analysis for an interface problem via minimax differentiability. Appl. Math. Comput., 219(12):6828-6842, 2013.

[4] H. Brezis. Analyse fonctionnelle. Collection Mathématiques Appliquées pour la Maîtrise. [Collection of Applied Mathematics for the Master's Degree]. Masson, Paris, 1983. Théorie et applications. [Theory and applications].

[5] A. Canelas, A. Laurain, and A. A. Novotny. A topological derivative-based method for the inverse potential problem. Journal of Computational Physics, (accepted for publication), 2013.

[6] R. Correa and A. Seeger. Directional derivative of a minimax function. Nonlinear Anal., 9(1):13-22, 1985.

[7] M. Costabel, M. Dauge, and S. Nicaise. Corner Singularities and Analytic Regularity for Linear Elliptic Systems. Part I: Smooth domains. 211 pages v2: Improvement of layout v2: Improvement of layout.

[8] M. C. Delfour and J.-P. Zolésio. Shape sensitivity analysis via min max differentiability. SIAM J. Control Optim., 26(4):834-862, 1988.

[9] M. C. Delfour and J.-P. Zolésio. Shapes and geometries, volume 4 of Advances in Design and Control. Society for Industrial and Applied Mathematics (SIAM), Philadelphia, PA, 2001.

[10] H. Egger, M. Hanke, C. Schneider, J. Schöberl, and S. Zaglmayr. Adjoint-based sampling methods for electromagnetic scattering. Inverse Problems, 26(7):074006, 17, 2010.

[11] I. Ekeland and R. Temam. Analyse convexe et problèmes variationnels. Dunod, 1974. Collection Études Mathématiques.

[12] S. Engleder and O. Steinbach. Boundary integral formulations for the forward problem in magnetic induction tomography. Mathematical Methods in the Applied Sciences, 34(9):1144-1156, 2011.

[13] M. Freiberger, A. Laurain, M. Hintermüller, A. Köstinger, and H. Scharfetter. Using the topological derivative for initializing a markov-chain monte carlo reconstruction in fluorescence tomography. In Proc. SPIE, volume 8088, 2011.

[14] M. Freiberger, A. Laurain, M. Hintermüller, and H. Scharfetter. Topological derivative for image reconstruction in fluorescence tomography. In Biomedizinische Technik/Biomedical Engineering., volume 55, page 1âĂŞ264, 2010.

[15] V. Girault and P. Raviart. Finite Element Methods for Navier-Stokes Equations. Springer-Verlag, Berlin, 1986.

[16] E. Giusti. Minimal surfaces and functions of bounded variation, volume 80 of Monographs in Mathematics. Birkhäuser Verlag, Basel, 1984.

[17] D. Gürsoy and H. Scharfetter. Optimum receiver array design for magnetic induction tomography. IEEE Trans. Biomed. Engineering, 56(5):1435-1441, 2009.

[18] L. He and S. Osher. Solving the chan-vese model by a multiphase level set algorithm based on the topological derivative. In Scale Space Variational Methods in Computer Vision. Lecture Notes in Computer Science, pages 777-788. Springer, Berlin, 2007.

[19] A. Henrot and M. Pierre. Variation et optimisation de formes, volume 48 of Mathématiques \& Applications (Berlin) [Mathematics \& Applications]. Springer, Berlin, 2005. Une analyse géométrique. [A geometric analysis].

[20] F. Hettlich. The domain derivative of time-harmonic electromagnetic waves at interfaces. Mathematical Methods in the Applied Sciences, 35(14):1681-1689, 2012.

[21] M. Hintermüller and A. Laurain. Electrical impedance tomography: from topology to shape. Control Cybernet., 37(4):913-933, 2008.

[22] M. Hintermüller and A. Laurain. Multiphase image segmentation and modulation recovery based on shape and topological sensitivity. J. Math. Imaging Vision, 35(1):1-22, 2009.

[23] M. Hintermüller, A. Laurain, and A. A. Novotny. Second-order topological expansion for electrical impedance tomography. Adv. Comput. Math., 36(2):235-265, 2012.

[24] M. Kolmbauer and U. Langer. A robust preconditioned minres solver for distributed time-periodic eddy current optimal control problems. SIAM Journal on Scientific Computing, 34(6):B785-B809, 2012

[25] A. Laurain, M. Hintermüller, M. Freiberger, and H. Scharfetter. Topological sensitivity analysis in fluorescence optical tomography. Inverse Problems, 29(2):025003, 30, 2013

[26] R. Merwa, K. Hollaus, B. Brandstätter, and H. Scharfetter. Numerical solution of the general 3d eddy current problem for 
magnetic induction tomography (spectroscopy). Physiological Measurement, 24(2):545, 2003.

[27] R. Merwa, K. Hollaus, P. Brunner, and H. Scharfetter. Solution of the inverse problem of magnetic induction tomography (mit). Physiological Measurement, 26(2):S241, 2005.

[28] P. Monk. Finite element methods for Maxwell's equations. Numerical Mathematics and Scientific Computation. Oxford University Press, New York, 2003.

[29] A. Novotny, A. Canelas, and A. Laurain. A new method for the inverse potential problem based on the topological derivative. In Z. D. Prado, editor, Proceedings of the XXXIV Iberian Latin-American Congress on Computational Methods in Engineering, ABMEC, Pirenopolis, GO, Brazil, November 2013.

[30] A. J. Peyton, Z. Z. Yu, G. Lyon, S. Al-Zeibak, J. Ferreira, J. Velez, F. Linhares, A. R. Borges, H. L. Xiong, N. H. Saunders, and M. S. Beck. An overview of electromagnetic inductance tomography: description of three different systems. Measurement Science and Technology, 7(3):261, 1996.

[31] H. Scharfetter, P. Brunner, and R. Merwa. Magnetic induction tomography: single-step solution of the 3-d inverse problem for differential image reconstruction. International Journal of Information and Systems Sciences, 2(4):585-606, 2006.

[32] H. Scharfetter, K. Hollaus, J. Rosell-Ferrer, and R. Merwa. Single-step 3-d image reconstruction in magnetic induction tomography: Theoretical limits of spatial resolution and contrast to noise ratio. Annals of Biomedical Engineering, 34(11):1786$1798,2006$.

[33] J. Sokołowski and J.-P. Zolésio. Introduction to shape optimization, volume 16 of Springer Series in Computational Mathematics. Springer-Verlag, Berlin, 1992. Shape sensitivity analysis.

[34] M. Soleimani, W. Lionheart, C. Riedel, and O. Dossel. Forward problem in 3d magnetic induction tomography (mit). In Proceedings of the 3rd World Congress on Industrial Process Tomography, pages 275-280, 2003.

[35] F. Tröltzsch and I. Yousept. PDE-constrained optimization of time-dependent 3D electromagnetic induction heating by alternating voltages. ESAIM Math. Model. Numer. Anal., 46(4):709-729, 2012.

[36] L. Vese and T. Chan. A multiphase level set framework for image segmentation using the mumford and shah model. Int. J. Comput. Vis., 50:271-293, 2002.

[37] H.-Y. Wei and M. Soleimani. Three-dimensional magnetic induction tomography imaging using a matrix free krylov subspace inversion algorithm. Progress In Electromagnetics Research (PIER), 122:29-45, 2012.

[38] H.-Y. Wei and M. Soleimani. Three-dimensional magnetic induction tomography imaging using a matrix free krylov subspace inversion algorithm. Progress In Electromagnetics Research, 122:29-45, 2012.

[39] H.-Y. Wei and M. Soleimani. Two-phase low conductivity flow imaging using magnetic induction tomography. Progress In Electromagnetics Research (PIER), 131:99-115, 2012.

[40] I. Yousept. Finite Element Analysis of an Optimal Control Problem in the Coefficients of Time-Harmonic Eddy Current Equations. Journal of Optimization Theory and Applications, 154(3):879-903, 2012.

[41] I. Yousept. Optimal control of Maxwell's equations with regularized state constraints. Computational Optimization and Applications, 52(2):559-581, 2012.

[42] I. Yousept. Optimal Control of Quasilinear $\boldsymbol{H}$ (curl)-Elliptic Partial Differential Equations in Magnetostatic Field Problems. SIAM J. Control Optim., 51(5):3624-3651, 2013. 